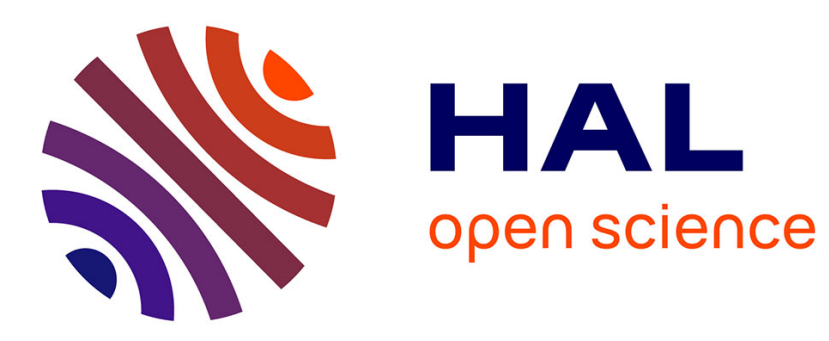

\title{
A new model of passive muscle tissue integrating Collagen Fibers: Consequences for muscle behavior analysis
}

\author{
Ali-Akbar Karkhaneh Yousefi, Mohammad Ali Nazari, Pascal Perrier, Masoud \\ Shariat Panahi, Yohan Payan
}

\section{To cite this version:}

Ali-Akbar Karkhaneh Yousefi, Mohammad Ali Nazari, Pascal Perrier, Masoud Shariat Panahi, Yohan Payan. A new model of passive muscle tissue integrating Collagen Fibers: Consequences for muscle behavior analysis. Journal of the mechanical behavior of biomedical materials, 2018, 88, pp.29-40. 10.1016/j.jmbbm.2018.07.042 . hal-01872331

\section{HAL Id: hal-01872331 \\ https://hal.science/hal-01872331}

Submitted on 12 Sep 2018

HAL is a multi-disciplinary open access archive for the deposit and dissemination of scientific research documents, whether they are published or not. The documents may come from teaching and research institutions in France or abroad, or from public or private research centers.
L'archive ouverte pluridisciplinaire HAL, est destinée au dépôt et à la diffusion de documents scientifiques de niveau recherche, publiés ou non, émanant des établissements d'enseignement et de recherche français ou étrangers, des laboratoires publics ou privés. 


\title{
A New Model of Passive Muscle Tissue Integrating Collagen Fibers: Consequences for Muscle Behavior Analysis
}

\author{
Ali-Akbar Karkhaneh Yousefi ${ }^{a 1}$, Mohammad Ali Nazari ${ }^{\text {a }}$, Pascal Perrier ${ }^{\text {, }}$ \\ Masoud Shariat Panahi ${ }^{\mathrm{a}}$, Yohan Payan ${ }^{\mathrm{c}}$ \\ ${ }^{a}$ School of Mechanical Engineering, College of Engineering, University of Tehran, Tehran, Iran \\ ${ }^{b}$ Univ. Grenoble Alpes, CNRS, Grenoble INP, Gipsa-lab, F38000 Grenoble France \\ ${ }^{c}$ Univ. Grenoble Alpes, CNRS, Grenoble INP, TIMC-IMAG, F38000 Grenoble France
}

\begin{abstract}
Mechanical properties of muscle tissue are crucial in biomechanical modeling of the human body. Muscle tissue is a combination of Muscle Fibers (MFs) and connective tissue including collagen and elastin fibers. There are a lot of passive muscle models in the literature but most of them do not consider any distinction between Collagen Fibers (CFs) and MFs, or at least do not consider the mechanical effects of the CFs on the Three-Dimensional (3-D) behavior of tissue. As a consequence, unfortunately, they cannot describe the observed stress-stretch behavior in tissue in which the reinforced direction is not parallel to the MF direction. In this research, a new passive muscle model is presented, in which the CFs are separately considered in the formulation: they are distributed along the MFs in a cross-shaped arrangement. Thanks to this new architecture, a mechanical reinforced direction can be proposed, in addition to the muscle main fiber direction.

The passive biomechanical properties of the genioglossus muscle of a bovine tongue have been measured under uniaxial tensile tests. To characterize the 3-D response of the tissue, tests have been performed in different directions with respect to the MF direction. Moreover, a Constitutive Law (CL) has been proposed for modeling this behavior. In addition to our measurements on the bovine genioglossus muscle, results published in the literature on experimental data from the longissimus dorsi of pigs and the chicken pectoralis muscle were used to appraise the applicability of the proposed model. It is demonstrated that the proposed passive muscle model provides an accurate description of the fiber-oriented nature of muscle tissue. Also, it has been shown that using Finite Element Analysis (FEA) it might be possible to predict the angle $\theta$ between CFs and MF.
\end{abstract}

Keywords: Muscle model; Passive behavior; Collagen fibers; Inverse FEA.

${ }^{1}$ Corresponding author: Ali.karkhaneh@gmail.com, Ali.karkhaneh@ut.ac.ir 


\section{Introduction}

Accounting properly the physical characteristics of the musculoskeletal system significantly affects the accuracy of computational models in predicting human body behavior in a variety of applications such as impact biomechanics, surgical simulations and tissue engineering (Takaza et al. 2013). Besides an accurate geometrical description, these models also need a comprehensive Constitutive Law (CL) to fully take into consideration the tissue material properties such as nonlinearity, viscoelasticity and anisotropy (Yousefi et al. 2018, Van Loocke et al. 2006, Gras et al. 2012, Morrow et al. 2010, Parente et al. 2009, Calvo et al. 2010 and Pioletti et al. 1998). In most of the studies, skeletal muscles have been considered as fiber-reinforced materials in which the stiffest direction is parallel to the Muscle Fiber (MF) direction (Morrow et al. 2010, Calvo et al. 2010, Martins et al. 1998, Humphrey and Yin 1987, Parente et al. 2009, Song et al. 2007 and Blemker et al. 2005). However, to the authors' knowledge, muscles have not been sufficiently investigated experimentally yet and the available CLs fail in describing their Three-Dimensional (3-D) passive behavior, especially for those that show less resistance in loading along the MF direction (Gindre et al. 2013, Nie et al. 2011 and Wheatley et al. 2016). Takaza and colleagues (2013) measured passive stress-stretch responses of the pig longissimus dorsi muscle under uniaxial tensile tests. The reported results show that the MF direction is the weakest direction in this muscle in terms of mechanical resistance to elongation. A more surprising behavior has been reported for the chicken pectoralis muscle in which the tissue is stiffer in the direction of $45^{\circ}$ with respect to the MF direction (Mohammadkhah et al. 2016).

Muscle tissue has frequently been considered as a network of MFs, Collagen Fibers (CFs), and elastin fibers embedded in an isotropic matrix (Hernández et al. 2011). The MFs are surrounded by connective tissue of three different anatomical properties made of CFs, which are from the most external to the most internal level: epimysium (surrounding the whole muscle), perimysium (surrounding fiber bundles), and endomysium (around each individual fiber) (Purslow 2010). The main course of the CFs determines one of the directions of material anisotropy and such fibers are mainly responsible for the passive behavior of the tissue. The active response, which is responsible for the contractile behavior, usually refers to the response of the muscular fibers (Grasa et al. 2016 and Gindre et al. 2013). 
In some long strap-like muscles, two parallel sets of wavy CFs in a crossed-ply arrangement have been observed in the epimysium (Purslow 1989 and 2010). Depending on the sarcomere length, the mean CF angle with respect to the MF axis varies in the range of $20^{\circ}$ to $80^{\circ}$. This explains why mechanical passive properties of some muscles are stronger in the direction transverse to the MF direction (Takaza et al. 2013, Mohammadkhah et al. 2016 and Wheatley et al. 2016). For these muscles, the available CLs, in which the CFs and the MFs are identified with the same direction, cannot describe the observed stress-stretch behavior. In these models, the existence of any reinforced direction other than the MF direction may impose some negative values on the anisotropic part of the stress tensor, which violates the convexity condition of the Strain Energy Function (SEF). To the authors knowledge, only for the cases in which the stiffest direction is perpendicular to the MF direction, the convexity condition is satisfied and it is possible to explain this behavior either using the invariants and structural tensors proposed by Schröder and Neff (2003) or assuming an ellipsoidal distribution of fibers in muscles in which the shortest diameter is along the direction of MFs (Wheatley et al. 2017). So, it seems that the effects of the MF and CFs and their respective directions have to be separately defined in the passive CLs of muscle tissue.

The tongue is one of the most intriguing human organs because it plays a vital role in respiration, suckling, gathering and manipulating food, swallowing, and speech. The mammalian tongue is a complex muscular structure, which is composed of the mucous membrane, intrinsic and extrinsic muscles, and connective tissue (Shall et al. 2012). Extrinsic muscles originate from bones external to the tongue and insert into the body of the tongue: the genioglossus, the hyoglossus, the styloglossus and the palatoglossus. These muscles are the main responsible for the large displacements and shaping of the tongue. Intrinsic muscles are fully embedded in the body of the tongue and determine more locally the shape of the tongue: the superior longitudinal, the inferior longitudinal, the verticalis and the transversalis (Gerard et al. 2006). Each of these muscles has some specific orientation dependent properties.

The human tongue has been widely investigated experimentally and numerically to describe its actions in the vocal tract (Rohan et al. 2017, Gerard et al. 2005 and 2006, Dang and Honda 2002, Vogt et al. 2006 and Fujita et al. 2007). Yousefi et al. (2018) proposed a visco-hyperelastic $\mathrm{CL}$ to describe the rate-dependent and anisotropic stress-stretch behavior in the superior longitudinal muscle and the mucous membrane of bovines. Finite element models intend to 
improve the understanding of the role of the tongue in speech production (Buchaillard et al. 2009) and swallowing (Stavness et al. 2006). Unfortunately, the complex behavior of the tongue tissue has been reduced by simplified models in previous researches. Due to the lack of experiments in tongue tissue, a passive CL considering specifically the nonlinear nature and the anisotropic behavior of each muscle has not been proposed yet.

In higher order mammals, the musculatures of tongues are similar (Gilbert et al. 2006 and Shall et al. 2012). It was therefore decided to focus this paper on bovine tongue tissue since it is still complicated to characterize the biomechanical properties of human tongue tissue. It is hypothesized that tongue tissue has similar passive constitutive behavior in humans and bovines. Obviously, the bovine tongue, which wraps around the grass and heaves to rip it off, has very specific abilities. We believe that this peculiarity can be explained by the size of the bovine tongue and by its capacity of producing an active force that is significantly higher than human tongue (Yousefi et al. 2018).

In this research, the mechanical properties of the genioglossus muscle of bovine tongue are investigated to propose a new passive mechanical model for muscle tissue, which can completely predict its fiber-oriented responses. To do this, the stress-stretch response of the bovine genioglossus muscle is measured under uniaxial tensile tests. Furthermore, a new CL is proposed for the passive behavior of muscle tissue which can specifically take into consideration the 3-D effects of the CFs. To evaluate the proposed model, its accuracy in approximating the experimental data is demonstrated for our data about the bovine genioglossus tissue as well as for published experimental data about the pig longissimus dorsi muscle (Takaza et al. 2013) and the chicken pectoralis muscle (Mohammadkhah et al. 2016).

The paper is organized in the following manner. In section 2, a new model of passive muscle behavior describing the nonlinear and orientation dependent response of muscle tissue is proposed. In section 3, the details of the bovine tongue sample preparation and test procedures are presented. In addition, the proposed model is used to approximate the stress-stretch response of the bovine tongue genioglossus muscle. The ability of this model in predicting the experimental data on the pig longissimus dorsi muscle and the chicken pectoralis muscle is examined in section 4. A discussion with perspectives closes this article. 


\section{A New Passive Muscle Model}

Most of the passive muscle models in the literature do not consider any distinction between CFs and MFs, or at least do not consider the mechanical effects of CFs on the 3-D behavior of tissue. These models cannot provide a realistic account of the observed stress-stretch responses in muscle tissue, especially in tissue that amazingly shows stronger resistance against elongation in a direction different from the MF alignment (Takaza et al. 2013, Hernández et al. 2011, Mohammadkhah et al. 2016 and Wheatley et al. 2016). Recently, Grasa et al. (2016) have introduced the CFs in their passive muscle model that can describe such an amazing stress-stretch behavior, but this model does not include the 3-D distribution of the CFs. Moreover, Wheatley et al. (2017) have proposed a model for muscles in which it is possible to predict that the stiffest direction can be perpendicular to the MFs direction. But their model uses a lot of material parameters and cannot explain the cases in which the stiffest direction is neither parallel to the MFs direction nor perpendicular to them (Mohammadkhah et al. 2016). From our point of view, to model the passive behavior of muscle tissue, it is essential to separately take into consideration the direction and action of the CFs, which are distinct from the ones of the MFs.

\subsection{Structure of the Muscle Tissue}

Regarding the approximately helical distribution of the CFs along each MF (Purslow 2010 and Gindre et al. 2013), we propose to consider them as a pair of crossed fibers in any plane containing the MFs as they can be approximately observed in practice in any cross-section parallel to the MF direction (Purslow 1989). Figure 1 schematically shows a slice of a muscle tissue and two arbitrary perpendicular planes P1 and P2. These planes are mechanically equivalent and contain the cross-shaped arrangement of CFs with angle $\theta$ with respect to the MF direction. Regardless of the dispersion of $\mathrm{CFs}$, angle $\theta$ represents the average direction of CFs in skeletal muscles. As depicted in figure 1, due to the symmetrical distribution of the CFs along the MFs, our model of the passive muscle tissue is similar to the transversely isotropic materials which show the same material properties in any direction in the isotropy plane which is perpendicular to the MF direction. But for $\theta \neq 0^{\circ}$, compared with the transversely isotropic materials, the reinforced direction is not necessarily either in the isotropy plane or the normal vector of the isotropy plane, namely the MF direction. Thus, the proposed model in figure 1 provides the possibility of the 
existence of some other direction with mechanical properties stronger than the ones in the direction of MFs.

Another significant effect of the CFs on the stress-stretch response, which has not been considered in the literature yet, is their 3-D nature as they are distributed in any plane containing the MFs. To characterize this, let us suppose that a muscle sample undergoes a uniaxial extension along the direction $X 1$ with acute angle $\phi$ with respect to the direction of the MFs, as depicted in figure 2. In general, there is a coordinate system $X 1 X 2 X 3$ which at least includes one of the symmetry planes of this sample. Such a plane contains $X 1$ and the MF direction as the CFs symmetrically surround it. Hence, there is no shear deformation out of this plane. Further, let $X 2$ be in the plane of symmetry and $X 3$ perpendicular to this plane.

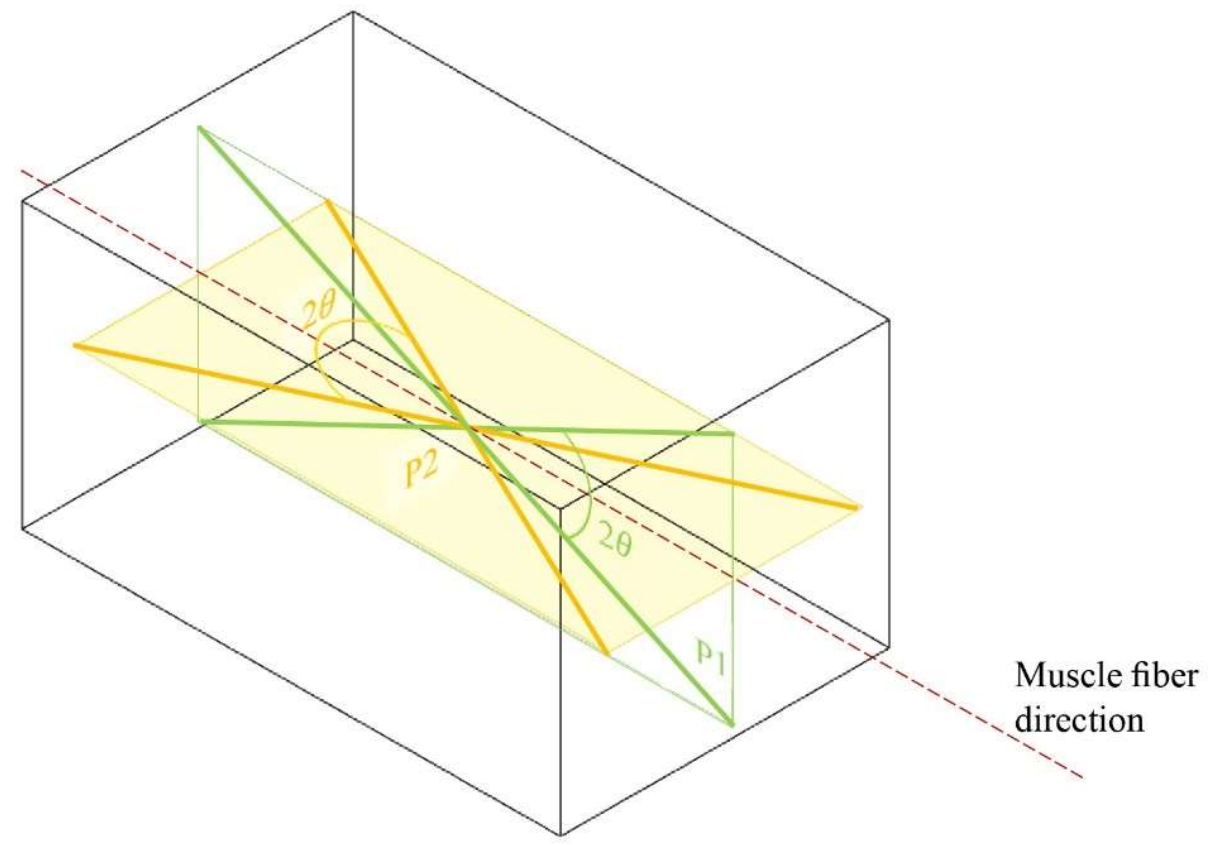

Figure 1. Schematic model of a muscle tissue in which a pair of cross-shaped CFs exists in any plane containing the MF direction.

In figure 2, as mentioned before, there are two mechanically equivalent $\mathrm{CFs}$ with unit vectors $\mathbf{a}_{1}=(\cos (\theta-\phi), \sin (\theta-\phi), 0)$ and $\mathbf{a}_{2}=(\cos (\theta+\phi),-\sin (\theta+\phi), 0)$ in the plane $X 1 X 2$. To measure the contribution of the other pairs of CFs to the stress response out of the $X 1 X 2$ plane, it is sufficient to consider the one which is located in the plane constructed with $X 3$ and the MF direction. Furthermore, it can easily be shown that these two families of CFs are along the unit vectors $\mathbf{g}_{1}=(\cos \theta \cos \phi,-\cos \theta \sin \phi, \sin \theta)$ and $\mathbf{g}_{2}=(\cos \theta \cos \phi,-\cos \theta \sin \phi,-\sin \theta)$. 


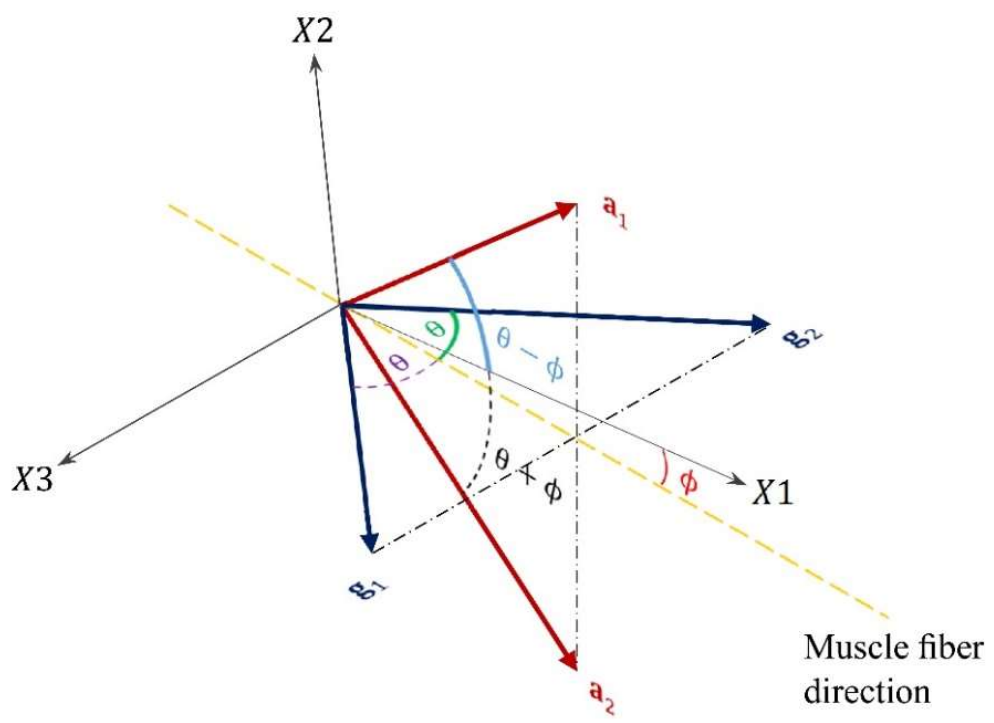

Figure 2. The configuration of the CFs and the MF direction in the coordinate system $X 1 X 2 X 3$.

\subsection{Kinematics and Stress Tensors}

A CL has to be chosen to describe the stress-stretch relationship of passive muscle tissue. Chagnon et al. (2015) reviewed and classified the most popular hyperelastic CLs for soft tissue. For a specific muscle tissue, based upon the material structure and shape of the measured stressstretch curves, an appropriate CL can be defined. To show how a stress-stretch response is related to a CL, let us briefly review the large deformation formulation in the framework of hyperelasticity. Let $\mathbf{X}$ and $\mathbf{x}$ be the position vector of material points in the reference (undeformed) and deformed configurations, respectively. For any function $\chi$ describing the motion $\mathbf{x}=\chi(\mathbf{X})$ which maps any position vector of the reference configuration to the deformed configuration, let $\mathbf{F}(\mathbf{X})=\partial \mathbf{x} / \partial \mathbf{X}$ be the deformation gradient tensor and $J=\operatorname{det} \mathbf{F}(\mathbf{X})>0$ the Jacobian representing the ratio of volume change during the deformation. Following Flory (1961), F can be decomposed as:

$$
\mathbf{F}=\left(J^{1 / 3} \mathbf{I}\right) \overline{\mathbf{F}}
$$

with a dilatational part $J^{1 / 3} \mathbf{I}$ and a distortional part $\overline{\mathbf{F}}$, in which $\mathbf{I}$ represents the second order identity tensor. The modified right Cauchy-Green strain tensor is given by $\overline{\mathbf{C}}=\overline{\mathbf{F}}^{\mathrm{T}} \overline{\mathbf{F}}$. For $3-\mathrm{D}$ hyperelastic CLs representing isotropic materials, the invariants of a strain tensor are used as variables to define a SEF in order to meet the requirement of objectivity. So, taking $\overline{\mathbf{C}}$ as this strain tensor, its two modified principal invariants are defined as: 


$$
\bar{I}_{1}=\operatorname{tr} \overline{\mathbf{C}}, \quad \bar{I}_{2}=\frac{1}{2}\left[(\operatorname{tr} \overline{\mathbf{C}})^{2}-\operatorname{tr}\left(\overline{\mathbf{C}}^{2}\right)\right]
$$

For anisotropic materials reinforced with two families of fibers, the modified principal invariants defined by equation (2) are not sufficient to describe the behavior. Hence, for such materials with two preferred fiber directions specified by the referential unit vectors $\mathbf{n}_{1}$ and $\mathbf{n}_{2}$, the symmetric structural tensors $\mathbf{A}_{1}$ and $\mathbf{A}_{2}$ are defined as:

$$
\begin{aligned}
& \mathbf{A}_{1}=\mathbf{n}_{1} \otimes \mathbf{n}_{1}, \quad \mathbf{A}_{2}=\mathbf{n}_{2} \otimes \mathbf{n}_{2} \\
& \bar{I}_{4 \mathbf{a}_{1}}=\operatorname{tr}\left(\overline{\mathbf{C}} \mathbf{A}_{1}\right) \\
& \bar{I}_{4 \mathbf{a}_{2}}=\operatorname{tr}\left(\overline{\mathbf{C}} \mathbf{A}_{2}\right)
\end{aligned}
$$

in which $\bar{I}_{4 \mathbf{a}_{1}}$ and $\bar{I}_{4 \mathbf{a}_{2}}$ are modified additional invariants (pseudo-invariants), which are the most common variables in defining the CLs for anisotropic materials (Spencer 1984). Also, $\otimes$ in equation (3a) represents the dyadic multiplication of two vectors.

In hyperelasticity, to describe the stress response for muscle tissue, it is postulated that a scalar-valued free energy function exists, which is called the Helmholtz free energy function $\psi$, also referred to as strain energy function or elastic potential energy function (Gasser et al. 2006). For isothermal processes, the thermodynamical principles reduce to the satisfaction of the Clausius-Duhem inequality

$$
\left(\mathbf{S}-2 \frac{\partial \psi}{\partial \mathbf{C}}\right): \frac{\dot{\mathbf{C}}}{2} \geq 0 \quad \forall \mathbf{C}, \dot{\mathbf{C}}
$$

where the operator ":" represents the double contraction of two tensors, $\mathbf{C}=J^{2 / 3} \overline{\mathbf{C}}$ represents the right Cauchy-Green strain tensor, $\dot{\mathbf{C}}$ is its material time derivative, and $\mathbf{S}$ is the second PiolaKirchhoff stress tensor. Without energy dissipations, $\mathbf{S}=2 \frac{\partial \psi}{\partial \mathbf{C}}$ can be a solution for equation (4).

\subsection{Constitutive Law}

To describe the stress-stretch response of the muscle tissue which is assumed to behave nearly incompressible, it was decided to propose the SEF $\psi$ in the decoupled form with a purely volumetric part $\psi_{v o l}$ as a measure of the required strain energy to change the volume and a purely isochoric part $\psi_{i s o}$ representing the stored strain energy by the distortion part of a deformation. The volumetric part is a function of $J$ and the isochoric part is defined as a function of the modified 
right Cauchy-Green strain tensor, the structural tensors constructed by the direction of the CFs and MFs, and the activation parameter $\alpha$ which determines the amount of the active stress in the muscle tissue. Therefore, in the general form, the SEF of the skeletal muscles can be stated as:

$$
\psi\left(\mathbf{C}, \mathbf{A}_{1}, \mathbf{A}_{2}, \mathbf{G}_{1}, \mathbf{G}_{2}, \mathbf{A}_{m f}, \alpha\right)=\psi_{v o l}(J)+\psi_{i s o}\left(\overline{\mathbf{C}}, \mathbf{A}_{1}, \mathbf{A}_{2}, \mathbf{G}_{1}, \mathbf{G}_{2}, \mathbf{A}_{m f}, \alpha\right)
$$

with the structural tensors $\mathbf{A}_{1}=\mathbf{a}_{1} \otimes \mathbf{a}_{1}, \mathbf{A}_{2}=\mathbf{a}_{2} \otimes \mathbf{a}_{2}, \mathbf{G}_{1}=\mathbf{g}_{1} \otimes \mathbf{g}_{1}$, and $\mathbf{G}_{2}=\mathbf{g}_{2} \otimes \mathbf{g}_{2}$ in which the unit vectors $\mathbf{a}_{1}, \mathbf{a}_{2}, \mathbf{g}_{1}$, and $\mathbf{g}_{2}$ have been introduced in figure 2. Also, $\mathbf{A}_{m f}=\mathbf{a}_{m f} \otimes \mathbf{a}_{m f}$ with the referential unit vector $\mathbf{a}_{m f}$ in the direction of the MFs. Equation (5) shows how the proposed model of this study distinguishes between the CFs and the MFs. In other words, $\mathbf{A}_{m f}$ is required to define to active response.

Moreover, the isochoric part of the SEF itself can be divided into the passive $\left(\psi_{p}\right)$ and active $\left(\psi_{a}\right)$ parts as:

$$
\psi_{i s o}\left(\overline{\mathbf{C}}, \mathbf{A}_{1}, \mathbf{A}_{2}, \mathbf{G}_{1}, \mathbf{G}_{2}, \mathbf{A}_{m f}, \alpha\right)=\psi_{p}\left(\overline{\mathbf{C}}, \mathbf{A}_{1}, \mathbf{A}_{2}, \mathbf{G}_{1}, \mathbf{G}_{2}\right)+\psi_{a}\left(\overline{\mathbf{C}}, \mathbf{A}_{m f}, \alpha\right)
$$

Since characterizing the passive behavior of the muscle tissue is the concern of this study, from now on the active part of the SEF is discarded. $\psi_{i s o}$ is generally defined by the invariants $\bar{I}_{1}$, $\bar{I}_{2}, J$, and $\bar{I}_{4}$. Hence, the SEF is proposed as:

$$
\begin{aligned}
& \psi\left(\bar{I}_{1}, \bar{I}_{2}, J, \bar{I}_{4}\right)=\psi_{\text {vol }}(J)+\psi_{\text {iso }}\left(\bar{I}_{1}, \bar{I}_{2}, \bar{I}_{4}\right) \\
& \psi_{\text {vol }}(J)=\frac{1}{2} k(J-1)^{2} \\
& \psi_{\text {iso }}\left(\bar{I}_{1}, \bar{I}_{2}, \bar{I}_{4}\right)=c_{1}\left(\bar{I}_{1}-3\right)^{2}+c_{2}\left(\bar{I}_{2}-3\right) \\
& +\frac{c_{3}}{c_{4}}\left\{\left(\sqrt{\bar{I}_{4 \mathbf{a}_{1}}}-1\right)^{c_{4}}+\left(\sqrt{\bar{I}_{4 \mathbf{a}_{2}}}-1\right)^{c_{4}}+\left(\sqrt{\bar{I}_{4 \mathbf{g}_{1}}}-1\right)^{c_{4}}\right. \\
& \left.+\left(\sqrt{\bar{I}_{4 \mathbf{g}_{2}}}-1\right)^{c_{4}}\right\}
\end{aligned}
$$

with the Lagrange multiplier $k$ and the material parameters $c_{1-4}$. All of the material parameters have to be non-negative to warrant the convexity condition of the SEF. Also, the modified fourth invariants $\bar{I}_{4 \mathbf{a}_{i}}$ and $\bar{I}_{4 \mathbf{g}_{i}}$ are equivalent to the square of the stretch along the unit vectors $\mathbf{a}_{\mathbf{i}}$ and $\mathbf{g}_{\mathbf{i}}$ scaled by $J^{-2 / 3}$, respectively. These invariants are separately considered in the proposed model to apply the 3-D effects of the CFs on the stress tensor. In equation ( $7 \mathrm{c})$, the first two terms are added to take into account the contribution of the matrix-fiber ensemble in an anisotropic material, and 
the third term characterizes the CFs resistance to elongation. Since all of the CFs have made of the same matter, they have the same mechanical properties and as a consequence, their contribution has been proposed by the same material parameters in equation $(7 \mathrm{c})$.

Although recent studies indicate that MFs and CFs have a contribution in compression (Van Loocke et al. 2006 and 2008 and Böl et al. 2014 and 2016), the overall resistance of muscle tissue in compression is a way lower than its resistance against tension (Mohammadkhah et al. 2016). Therefore, since the concern of this study is to approximate the stress-stretch response of muscle tissue in tension, the influence of the resistance of the CFs in compression was considered to be negligible. In other words, for $\bar{I}_{4 \mathbf{a}_{i}}<1$ and $\bar{I}_{4 \mathbf{g}_{i}}<1$, the corresponding anisotropic terms in equation (7c) are considered to be negligible.

For the proposed model in equations (7), $\mathbf{S}$ can be defined in the decoupled form too (Holzapfel 2000). In this manner, $\mathbf{S}$ is given by:

$$
\begin{aligned}
& \mathbf{S}=2 \frac{\partial \psi}{\partial \mathbf{C}}=\mathbf{S}_{v o l}+\mathbf{S}_{i s o} \\
& \mathbf{S}_{v o l}=k J(J-1) \mathbf{C}^{-1} \\
& \mathbf{S}_{i s o}=J^{-2 / 3}\left(\overline{\mathbf{S}}-\frac{1}{3} \operatorname{tr}(\overline{\mathbf{S}} \mathbf{C}) \mathbf{C}^{-1}\right) \\
& \overline{\mathbf{S}}=2 \frac{\partial \psi_{i s o}}{\partial \overline{\mathbf{C}}}=4 c_{1}\left(\bar{I}_{1}-3\right) \mathbf{I}+2 c_{2}\left(\bar{I}_{1} \mathbf{I}-\overline{\mathbf{C}}\right) \\
& +c_{3}\left\{\frac{\left(\sqrt{\bar{I}_{4 \mathbf{a}_{1}}}-1\right)^{\left(c_{4}-1\right)}}{\sqrt{\bar{I}_{4 \mathbf{a}_{1}}}} \mathbf{A}_{1}+\frac{\left(\sqrt{\bar{I}_{4 \mathbf{a}_{2}}}-1\right)^{\left(c_{4}-1\right)}}{\sqrt{\bar{I}_{4 \mathbf{a}_{2}}}} \mathbf{A}_{2}\right. \\
& \left.+\frac{\left(\sqrt{\bar{I}_{4 \mathbf{g}_{1}}}-1\right)^{\left(c_{4}-1\right)}}{\sqrt{\bar{I}_{4 \mathbf{g}_{1}}}} \quad \mathbf{G}_{1}+\frac{\left(\sqrt{\bar{I}_{4 \mathbf{g}_{2}}}-1\right)^{\left(c_{4}-1\right)}}{\sqrt{\bar{I}_{4 \mathbf{g}_{2}}}} \quad \mathbf{G}_{2}\right\}
\end{aligned}
$$

where $\overline{\mathbf{S}}$ is called the fictitious second Piola-Kirchhoff stress tensor. See Holzapfel (2000) for calculating the required derivatives in writing equation (8) and more detailed explanations of extracting equation $(8 \mathrm{c})$. 
Generally, when a muscle tissue is loaded along the $X 1$ direction as depicted in figure 2, F can be written as:

$$
\mathbf{F}=\left[\begin{array}{ccc}
F_{11} & F_{12} & 0 \\
F_{21} & F_{22} & 0 \\
0 & 0 & F_{33}
\end{array}\right]
$$

in which the off-diagonal components vanish only for $\phi=0^{\circ}$ and $\phi=90^{\circ} ; F_{33}=$ $1 /\left(F_{11} F_{22}-F_{12} F_{21}\right)$ due to the near incompressibility assumption. Thus, using the relationship $\mathbf{P}=\mathbf{F S}$, from equations (8) and (9), the components of the nominal stress tensor $\mathbf{P}$ can be obtained as a function of the components of $\mathbf{F}$. In general, it is not possible to determine all the components of $\mathbf{F}$ for an arbitrary $\phi$. For $\phi \neq 0^{\circ}$ sample undergoes shear stress in the $X 1 X 2$ plane and for $\phi=90^{\circ}$ equality of $F_{22}$ and $F_{33}$ depends on the value of $\theta$. Therefore, there is no explicit relationship between the stress and the controllable parameters of the machine. Thus, an inverse finite element method has to be used in order to determine the uncontrollable components of $\mathbf{F}$ from the experimental data.

\subsection{Adjustment of Material Parameters}

The material parameters can be determined by adjusting the nominal stress-stretch relationship in order to fit the experimental data points at $\phi=0^{\circ}$ and $\phi=90^{\circ}$. Since $\mathbf{P}$ in the case of $\phi=90^{\circ}$ is not necessarily only a function of the controllable parameters of the machine, some complementary tools like an Finite Element Analysis (FEA) have to be employed to determine the uncontrollable components of $\mathbf{F}$. To do this, a first guess of the values of the material parameters is inferred from the results of tensile tests at $\phi=0^{\circ}$ and $\phi=90^{\circ}$ with a MATLAB script based on the Genetic optimization algorithm (Goldberg 2006), in which $\mathbf{F}$ is assumed to be symmetric in the directions $X 2$ and $X 3$, which enables to simplify the stress-stretch relationship to a function of stretch $F_{11}$. In other words, the material parameters are estimated by minimizing the objective function $f\left(c_{i}\right)$ which represents a measure of the error between the experimental stress and the model prediction as:

$$
f\left(c_{i}\right)=\frac{1}{m} \sum_{i=1}^{m}\left(P_{11 \exp }^{i}-P_{11 \text { pre }}^{i}\right)_{\phi=0^{\circ}}^{2}+\frac{1}{n} \sum_{j=1}^{n}\left(P_{11 \exp }^{j}-P_{11 \text { pre }}^{j}\right)_{\phi=90^{\circ}}^{2}
$$


in which $P_{11 \exp }$ and $P_{11 \text { pre }}$ are the experimental and predicted nominal stress values along the direction $X 1$, respectively. Also, $m$ and $n$ are the number of data points for the cases of $\phi=0^{\circ}$ and $90^{\circ}$.

Then, the tensile test with $\phi=90^{\circ}$ is simulated with ABAQUS explicit solver by implementing a user-defined material model in subroutine VUMAT. After the FEA, changes of the non-zero components of $\mathbf{F}$ during the loading time are ready to be used in $\mathbf{P}$. Thus, updated values of the material parameters can be calculated through the optimization process using equation (10), in which $\mathbf{F}$ is taken from the FEA. The proposed procedure of the material parameters estimation has to be iterated with the updated parameters to converge to constant values. Figure 3 shows the required steps to calculate the material parameters $c_{i}$ of our proposed CL via uniaxial tensile tests.

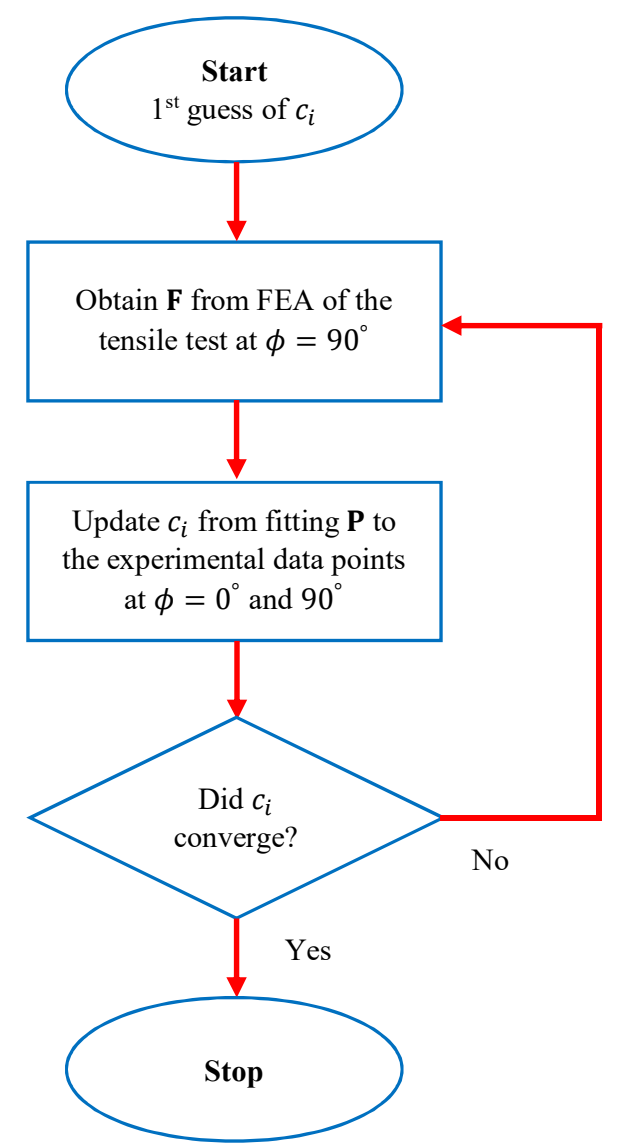

Figure 3. Flowchart of the material parameters estimation procedure for muscle tissue via uniaxial tensile tests. 


\section{Bovine Tongue Genioglossus Muscle}

In this section, the mechanical properties of the genioglossus muscle of bovine tongue are investigated under uniaxial tensile tests. Furthermore, the proposed CL in equations (7) is evaluated by approximating the measured stress-stretch responses of this muscle.

\subsection{Experimental Tensile Tests}

In order to accurately measure the mechanical properties of the bovine tongue tissue, three freshly slaughtered adult bovine tongues were used. Immediately after the sacrifice done early morning, the tongue was cut from the larynx and immersed in a saline solution at $4^{\circ} \mathrm{C}$ to keep it fresh and to prevent it from degradation (Hernández et al. 2011 and Gras et al. 2012). Due to the complex structure and the direction variation of tongue fibers (Gilbert et al. 2006), cutting an appropriate sample with a fixed fiber direction from each muscle part is difficult in practice. So in the current research, the samples were cut from the genioglossus tissue which is the largest muscle of the tongue. The ratio of the grip-to-grip length to width of the samples was set at least around 7 which was recommended by Hernández-Gascón et al. (2014). Besides, finite element results show that such long ratio is sufficient to achieve a uniform stretch distribution in the central area of a sample even in the presence of the anisotropy and shear deformations. These samples underwent a uniaxial tensile test on a Santam STM-1 machine with a $6 \mathrm{~kg}$ full-scale load cell. All of the reported data in this paper have been averaged among the measured stress-stretch behavior in different samples dissected from three bovine tongues. Figure 4 shows the bovine tongue and a corresponding sample during a tensile test.

The genioglossus originates on the genial tubercle on the inside of the anterior mandible and inserts on the ventromedial base of the tongue. It can therefore pull the tongue toward the mandible and as a result of tissue incompressibility protrude the tongue outside the mouth (Gerard et al. 2006). We assume that the response of the genioglossus tissue to the uniaxial tensile tests is strongly dependent on the angle between the CFs direction and the tensile force. To examine this dependency, some rectangular shape samples were dissected from the genioglossus with angles $90^{\circ}, 45^{\circ}$, and $0^{\circ}$ with respect to the MF alignment. Six samples along the MF direction, five samples at $45^{\circ}$, and five samples at $90^{\circ}$ were prepared to measure the average response in the genioglossus tissue. Although there are some difficulties in dissecting samples from muscle tissue, it was tried to cut them in a rectangular shape as much as possible since geometrical imperfections 
may adversely affect the accuracy of the identified material parameters (Böl et al. 2012). Tests were performed at the speed of $2 \mathrm{~mm} / \mathrm{min}$ to measure only the quasi-static response of the tissue. Table 1 presents the number of samples dissected from the bovine genioglossus muscle and their mean geometrical dimensions as well as the standard deviation values (sdv).

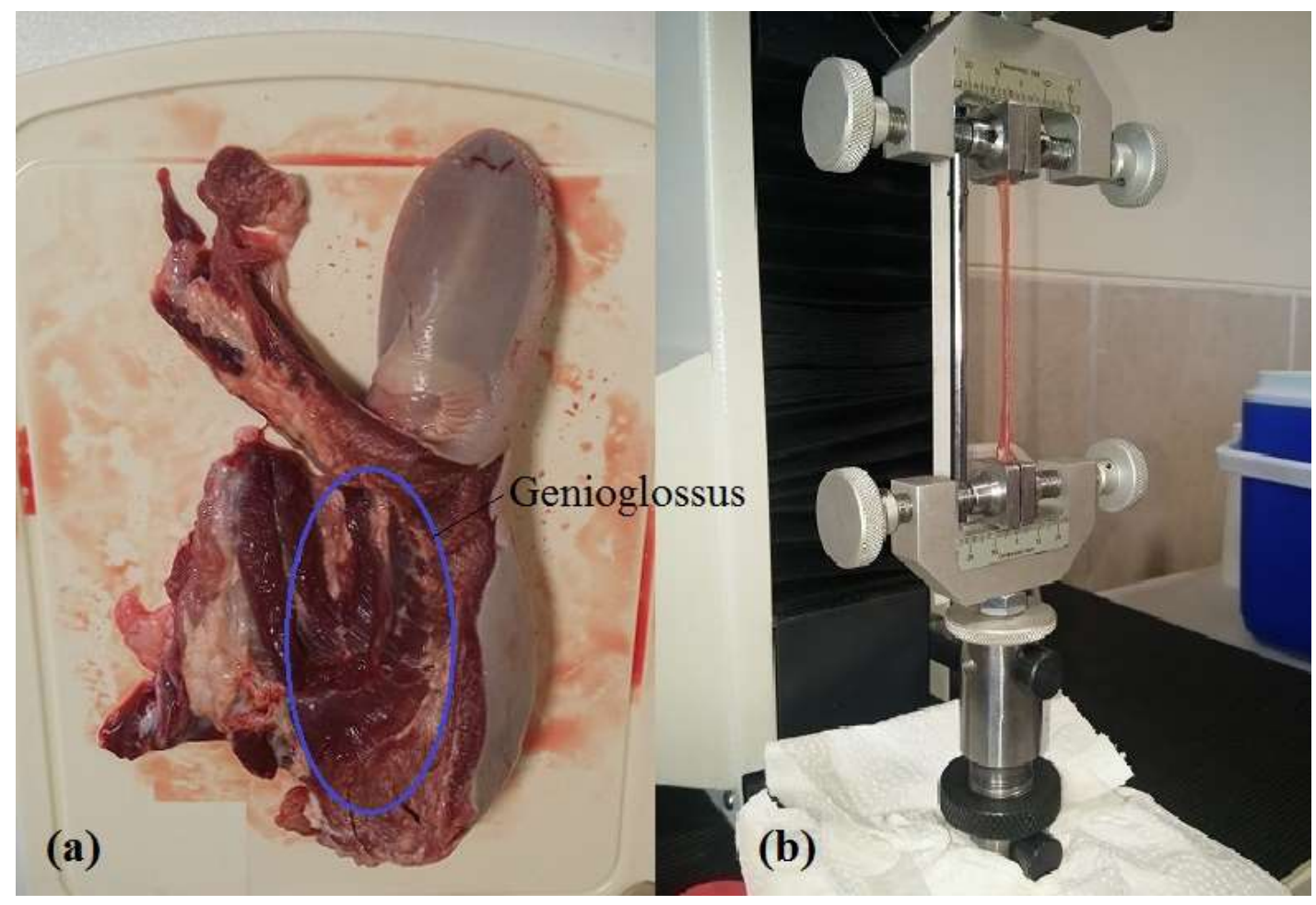

Figure 4. Bovine tongue (a) and a sample of bovine tongue muscle under uniaxial tension test (b).

Table1. Geometrical information of the samples dissected from the bovine genioglossus muscle.

\begin{tabular}{|c|c|c|c|c|}
\hline $\begin{array}{c}\text { Orientation to the } \\
\text { muscle fibers }\end{array}$ & $\begin{array}{c}\text { Number of } \\
\text { samples (-) }\end{array}$ & $\begin{array}{c}\text { Average } \\
\text { length }{ }^{*} \pm \text { std (mm) }\end{array}$ & $\begin{array}{c}\text { Average } \\
\text { width } \pm \text { std (mm) }\end{array}$ & $\begin{array}{c}\text { Average } \\
\text { height } \pm \text { std (mm) }\end{array}$ \\
\hline$\phi=0^{\circ}$ & 6 & $49.0 \pm 2.7$ & $7.1 \pm 0.2$ & $6.5 \pm 0.3$ \\
\hline$\phi=45^{\circ}$ & 5 & $45.1 \pm 3.1$ & $6.6 \pm 0.3$ & $6.3 \pm 0.2$ \\
\hline$\phi=90^{\circ}$ & 5 & $42.3 \pm 1.9$ & $6.0 \pm 0.3$ & $5.8 \pm 0.4$ \\
\hline
\end{tabular}

"Length means the distance between the grips before starting a test, it does not include the gripped parts of the samples.

Also, the stretch ratio has been measured by dividing the instantaneous distance between the grips to the initial one. Of course there are some optical techniques to measure the stretch ratio in the middle section of a sample, but the differences between these two methods remain less than $1 \%$ even after applying 50\% of stretch (Tian et al. 2015). After fastening the grips, the amount of the gripped length between the platens was controlled by a marker line on the platens as an 
indicator to show whether slippage occurred or not. Then, tests started and continued until rupture and the stress-stretch curves have been reported up until a considerable decrease in the load cell. To prevent the damage caused by fastening the grips and increase the friction between the muscle and platens, a particular glue named mitreapel was used just in those areas. This method considerably facilitates obtaining smoother stress-stretch curves to higher ranges of stretch.

\subsection{Model Predictions}

Experimental tests from samples of the genioglossus muscle have been performed at $\phi=$ $0^{\circ}, 45^{\circ}$, and $90^{\circ}$. Although the angle $\theta$ between CFs and MFs has not been experimentally measured, it seems to be possible to estimate this angle thanks to FEA, since $\theta$ is a parameter of this analysis. To do this, the material parameters have been determined for different values of $\theta$; then the accuracy of the prediction of each set of $\theta$ has been examined. As the genioglossus tissue is stronger when it is loaded perpendicular to the MFs direction, the searching space of angle $\theta$ has been limited to values greater than $45^{\circ}$. Following the material parameters estimation procedure described in figure $3, c_{1-4}$ have been accurately determined for some arbitrary values of $\theta=60^{\circ}, 63^{\circ}, 65^{\circ}$, and $70^{\circ}$. For instance, in the case of $\theta=65^{\circ}$, figure 5 shows the nominal stress-stretch responses of the proposed model in equations (7) fitted to the experimental data points at $\phi=0^{\circ}$ and $90^{\circ}$. The standard deviation curves around the mean values are also shown in gray lines. In this figure, the coefficient of determination $R^{2}$, provides a measure of goodness of fitting, shows that the material parameters are well-defined to fit the experimental points. The corresponding estimated values of $c_{1-4}$ and $k$ are provided in table 2. Also, in table 2 the goodness of fitting of the proposed model for each set of $\theta$ has been provided. The large value of $k$ ensures the incompressibility of material. Moreover, a convergence study to show that the final values of the material parameters are independent of a first guess has been presented in appendix A.

Table 2. Material parameters of the bovine genioglossus muscle tissue estimated for the proposed model.

\begin{tabular}{|c|c|c|c|c|c|c|c|}
\hline & $\begin{array}{c}c_{1} \\
(\mathrm{kPa})\end{array}$ & $\begin{array}{c}c_{2} \\
(\mathrm{kPa})\end{array}$ & $\begin{array}{c}c_{3} \\
(\mathrm{kPa})\end{array}$ & $\begin{array}{l}C_{4} \\
(-)\end{array}$ & $\begin{array}{c}k \\
(\mathrm{kPa})\end{array}$ & $\begin{array}{l}R^{2} \text { for } \\
\phi=0^{\circ}\end{array}$ & $\begin{array}{c}R^{2} \text { for } \\
\phi=90^{\circ}\end{array}$ \\
\hline$\theta=60^{\circ}$ & 9.668 & 7.369 & 373.012 & 2.379 & \multirow{4}{*}{$1 \mathrm{E} 7$} & 0.992 & 0.996 \\
\hline$\theta=63^{\circ}$ & 10.566 & 7.034 & 328.219 & 2.419 & & 0.994 & 0.999 \\
\hline$\theta=65^{\circ}$ & 10.566 & 7.034 & 313.181 & 2.462 & & 0.994 & 0.999 \\
\hline$\theta=70^{\circ}$ & 10.566 & 7.034 & 278.269 & 2.534 & & 0.994 & 0.999 \\
\hline
\end{tabular}




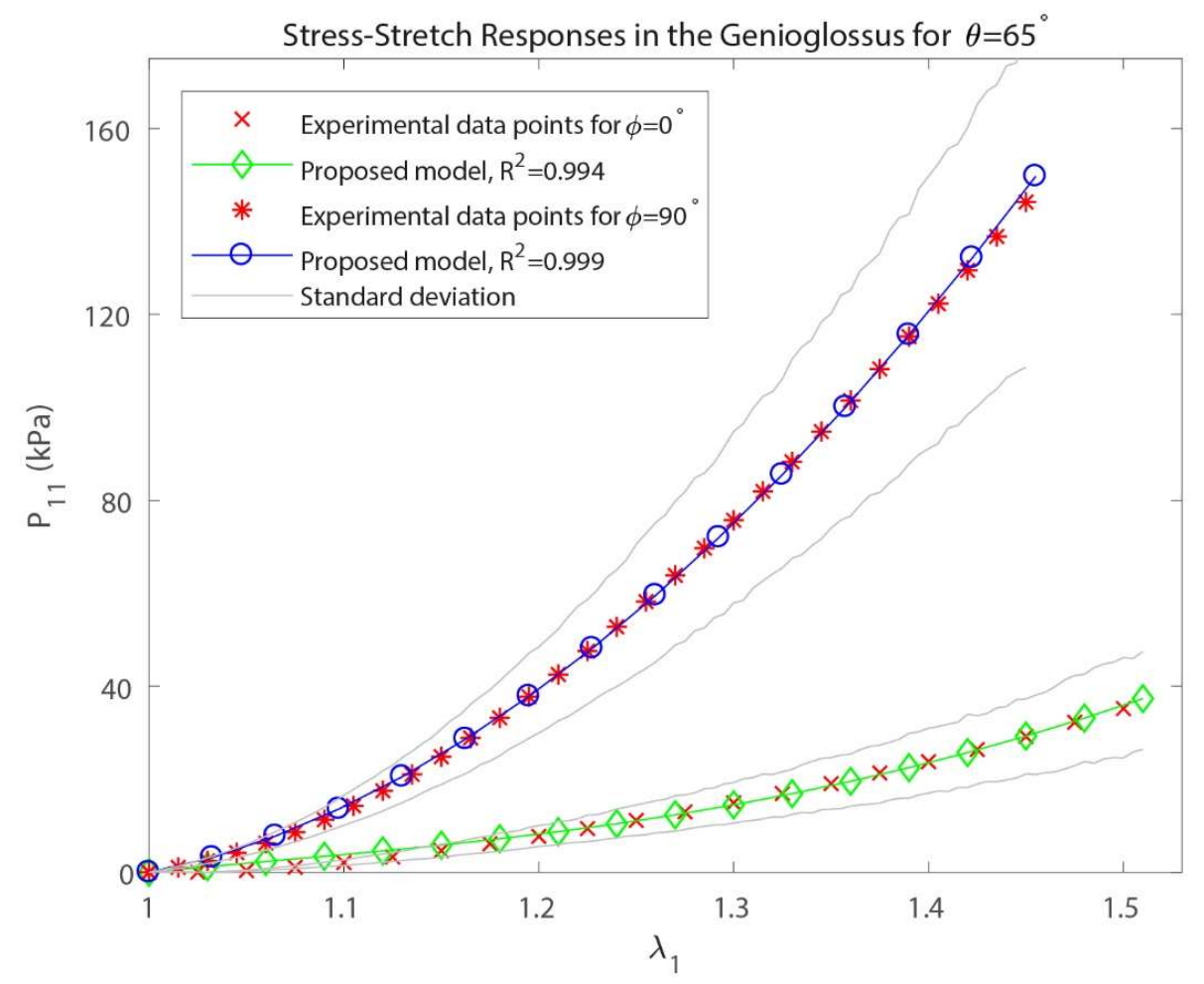

Figure 5. Stress-stretch responses of the bovine genioglossus muscle tissue to the uniaxial tension tests in different loading angles with respect to the MF direction. Also, the best approximation of the proposed model is plotted.

For the cases of $\theta>63^{\circ}$, when the genioglossus muscle is extended parallel to the MFs direction, the CFs have no contribution to bearing the tensile load. Although the angle $\theta$ has changed, the parameters $c_{1}$ and $c_{2}$ remained constant in table 2 . It can be explained by the fact that for those values of $\theta$, CFs do not undergo any stretch over the reported ranges of stretch in figure 5. Theoretically and physically, of course they can be recruited but beyond the reported ranges of stretch. Moreover, $c_{3}$ and $c_{4}$ vary as $\theta$ increases since the CFs become a more contributor to the overall response when the muscle is loaded perpendicular to the MFs direction.

Using these estimated material parameters, the accuracy of the proposed model in equations (7) has been examined at $\phi=45^{\circ}$. In this case, the nominal stress-stretch relationship is plotted in figure 6 together with the standard deviation curves. The proposed model has been implemented in the ABAQUS explicit solver. Nominal stresses for $\theta=60^{\circ}, 63^{\circ}, 65^{\circ}$, and $70^{\circ}$ at the most central point of the sample are plotted in figure 6 . As can be seen from this figure, the proposed passive muscle model with the tuned material parameters for $\theta=63^{\circ}$ accurately predicts the behavior of 
the genioglossus muscle. So, it seems that the mean angle between CFs and MFs in the reference configuration might be very close to $\theta=63^{\circ}$ for the genioglossus tissue.

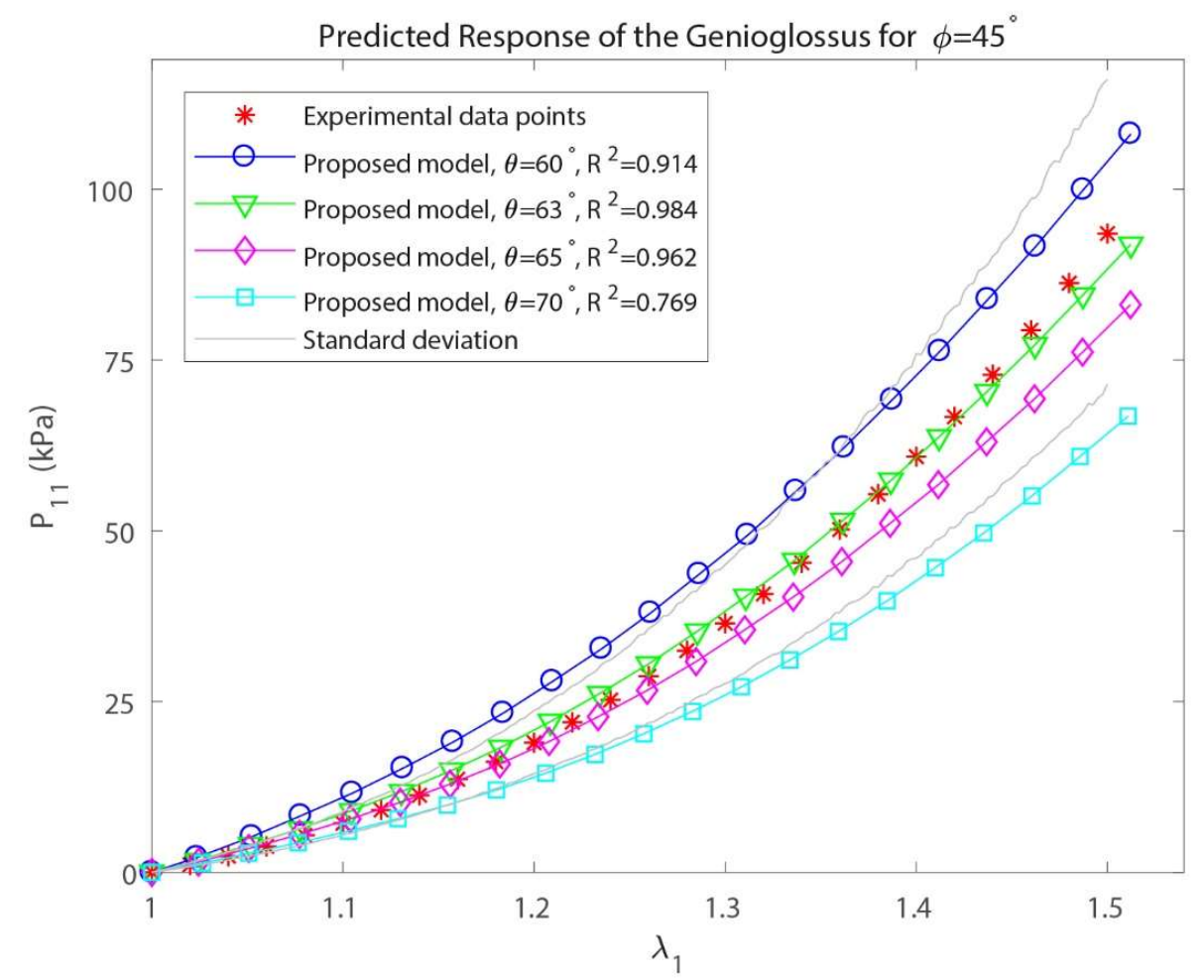

Figure 6. Comparison of the accuracy of the stress-stretch prediction of the proposed model for the bovine genioglossus muscle tissue in loading at different angles $\theta$.

In order to provide a better understanding of the tensile behavior of muscles predicted by the proposed model, the distribution of the nominal stress $P_{11}$ in each loading direction is shown in figure 7. To do this, using the estimated material parameters for the case of $\theta=63^{\circ}, 3$ samples with the same mesh and geometrical dimensions $7 \mathrm{~mm} \times 7 \mathrm{~mm} \times 49 \mathrm{~mm}$ underwent a $40 \%$ increase in the total length. To apply the boundary conditions, the bottom surface of samples with the normal vector $\mathrm{x}$ was pinned and the top surface was subjected to $40 \%$ of an increase in length along the $\mathrm{x}$-direction. Moreover, the top surface was fixed in the directions of $\mathrm{y}$ and $\mathrm{z}$ (Gasser et al. 2006). Figure (7b) shows that even in the presence of shear deformations caused by the nonsymmetrical distribution of the CFs along the loading direction, the length to width ratio of 7 is sufficient to filter the gripping effects and provide a uniform stress distribution in the middle area. 

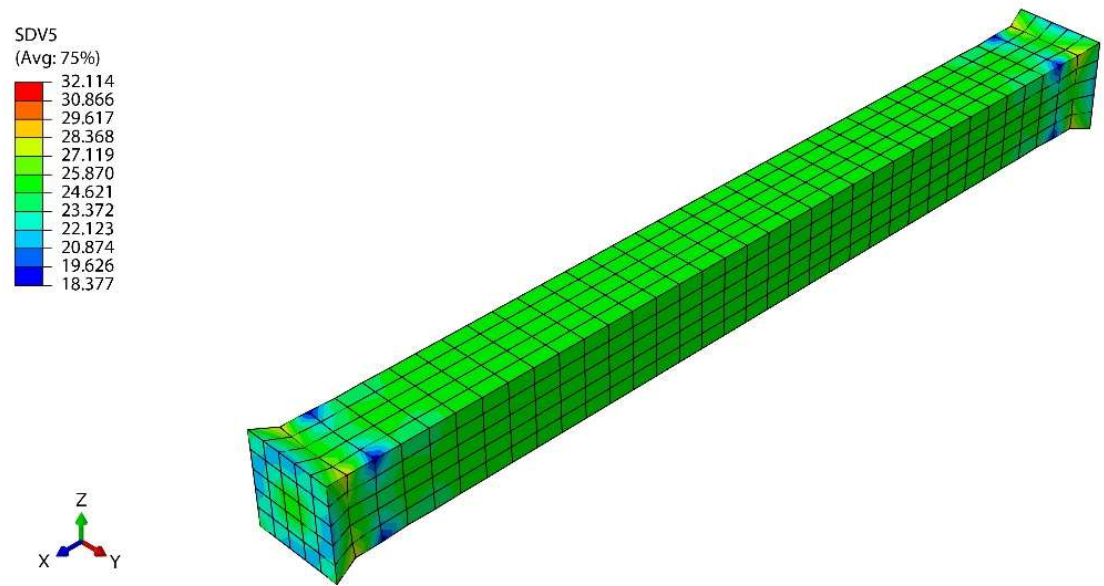

(a)
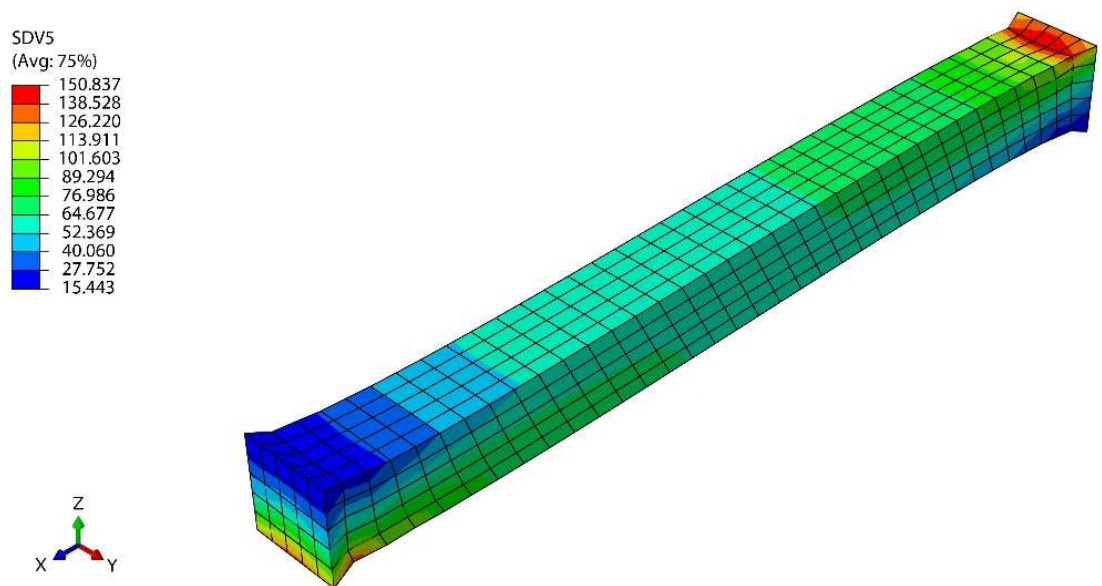

(b)
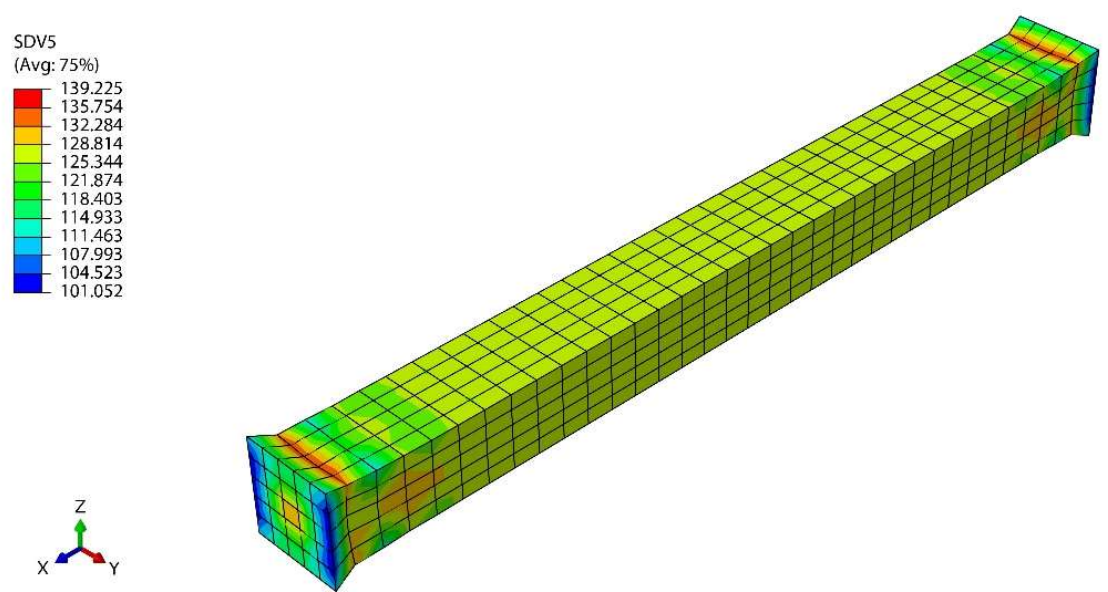

(c)

Figure 7. Distribution of the nominal stress $P_{11}$ after applying $40 \%$ of stretch to the total length in the genioglossus muscle for $\theta=63^{\circ}$. (a) is for $\phi=0^{\circ}$ and muscle fibers are along the x-direction, in (b) the direction of muscle fibers makes angle $\phi=45^{\circ}$ with the $\mathrm{x}$-direction in the plane $\mathrm{xz}$, and in (c) muscle fibers are along the z-direction which means $\phi=90^{\circ}$. 
Moreover, for the case of $\phi=90^{\circ}$ in figure 7 , the transversal stretch ratios $\lambda_{y}$ and $\lambda_{z}$ are not equivalent which is in agreement with some experimental findings (Takaza et al. 2013 and Mohammadkhah et al. 2016). To be more specific, when a sample is loaded in a direction perpendicular to the direction of the muscle fibers, in the transverse plane there will be a higher amount of shortening along the muscle fibers. The authors guess that there are two contributors to this behavior. First, in such condition, the stretched collagen fibers in the plane xz have a compressive component of stress along the muscle fibers. Consequently, the tissue is forced into a biased shortening in that direction. Second, it seemed that the existence of the cross-bridges is something that allows the actin and myosin filaments to have more spaces for shortening even in the passive state. For instance, figure 8 shows the initial and deformed shape of the cross-section in the middle section of samples shown in figure 7. As depicted in figure 8 for the case of $\phi=90^{\circ}$, the sample underwent a higher amount of shortening in the z-direction which is parallel to the muscle fibers.

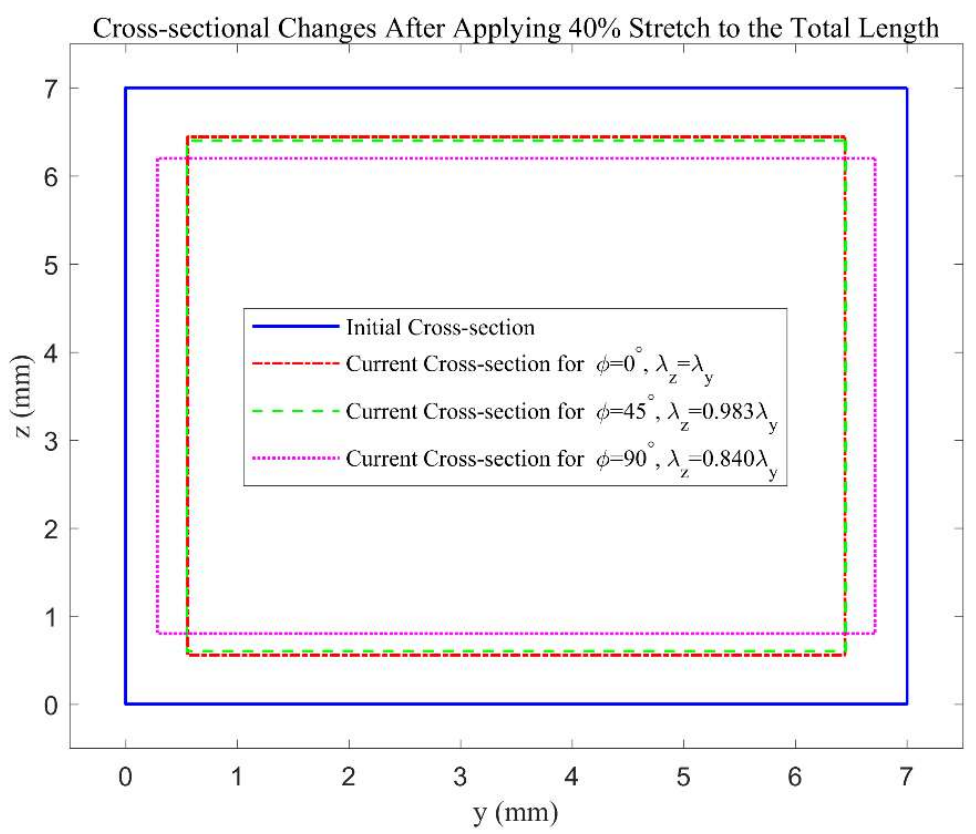

Figure 8. Changes in the middle cross-section after applying $40 \%$ increase to the total length ( $\mathrm{x}$ direction) in the genioglossus muscle. In the case of $\phi=0^{\circ}$, there is a symmetric deformation in the transversal directions $y$ and $z$. In the case of $\phi=45^{\circ}$ in which the muscle fibers are in the plane $x z$, the amount of shear deformation is a parameter that affects the values of the transversal stretches. Also, for $\phi=90^{\circ}$, the muscle fibers are along the $\mathrm{z}$-direction. 


\section{Model Evaluation on Literature Data}

To provide a broader evaluation of our CL, we propose to confront it with experimental data describing the elastic responses of the pig longissimus dorsi muscle tissue (Takaza et al. 2013 and Gindre et al. 2013) and the chicken pectoralis muscle (Mohammadkhah et al. 2016).

\subsection{Pig Longissimus Dorsi Muscle}

The experimental stress-stretch responses of the pig longissimus dorsi muscle were measured at $\phi=0^{\circ}, 30^{\circ}, 45^{\circ}, 60^{\circ}$ and $90^{\circ}$ by Takaza et al. (2013). Results show that not only the tissue becomes stronger by increasing the loading angle $\phi$ but also the curvature of the stressstretch curve changes. So, it is difficult to find a CL which can smoothly describe this curvature variation with a few number of material parameters. These experimental measurements suggest that the CFs have more contribution to the resultant response when the tissue is loaded at $\phi=90^{\circ}$ than at $\phi=0^{\circ}$. So, the angle $\theta$ must be greater than $45^{\circ}$. Similar to the method used for the bovine genioglossus muscle tissue, $c_{1-4}$ have been determined for different values of $\theta$. In this case, the Cauchy stress tensor $\boldsymbol{\sigma}=J^{-1} \mathbf{F S} \mathbf{F}^{T}$ which has been used by Takaza to report the behavior of the longissimus dorsi muscle can be computed from equations (8) and (9). Then, $c_{1-4}$ have been estimated by fitting the Cauchy stress-stretch relationship to the experimental measurements at $\phi=0^{\circ}$ and $90^{\circ}$ according to the procedure proposed in figure 3 . The estimated values of the parameters $c_{1-4}$ for $\theta=59^{\circ}, 63^{\circ}$, and $65^{\circ}$ are given in table 3 . Figure 9 shows the range of the approximated Cauchy stress in the proposed model for these values of $\theta$.

Table 3. Material parameters of the pig longissimus dorsi muscle tissue estimated for the proposed model.

\begin{tabular}{|c|c|c|c|c|c|c|c|}
\hline & $\begin{array}{c}c_{1} \\
(\mathrm{kPa})\end{array}$ & $\begin{array}{c}c_{2} \\
(\mathrm{kPa})\end{array}$ & $\begin{array}{c}c_{3} \\
(\mathrm{kPa})\end{array}$ & $\begin{array}{l}c_{4} \\
(-)\end{array}$ & $\begin{array}{c}k \\
(\mathrm{kPa})\end{array}$ & $\begin{array}{l}R^{2} \text { for } \\
\phi=0^{\circ}\end{array}$ & $\begin{array}{c}R^{2} \text { for } \\
\phi=90^{\circ}\end{array}$ \\
\hline$\theta=59^{\circ}$ & 89.451 & 12.194 & 220.296 & 1.511 & \multirow{3}{*}{$1 \mathrm{E} 7$} & 0.998 & 0.977 \\
\hline$\theta=63^{\circ}$ & 98.736 & 11.073 & 251.194 & 1.637 & & 0.999 & 0.986 \\
\hline$\theta=65^{\circ}$ & 98.750 & 11.067 & 216.285 & 1.630 & & 0.999 & 0.993 \\
\hline
\end{tabular}




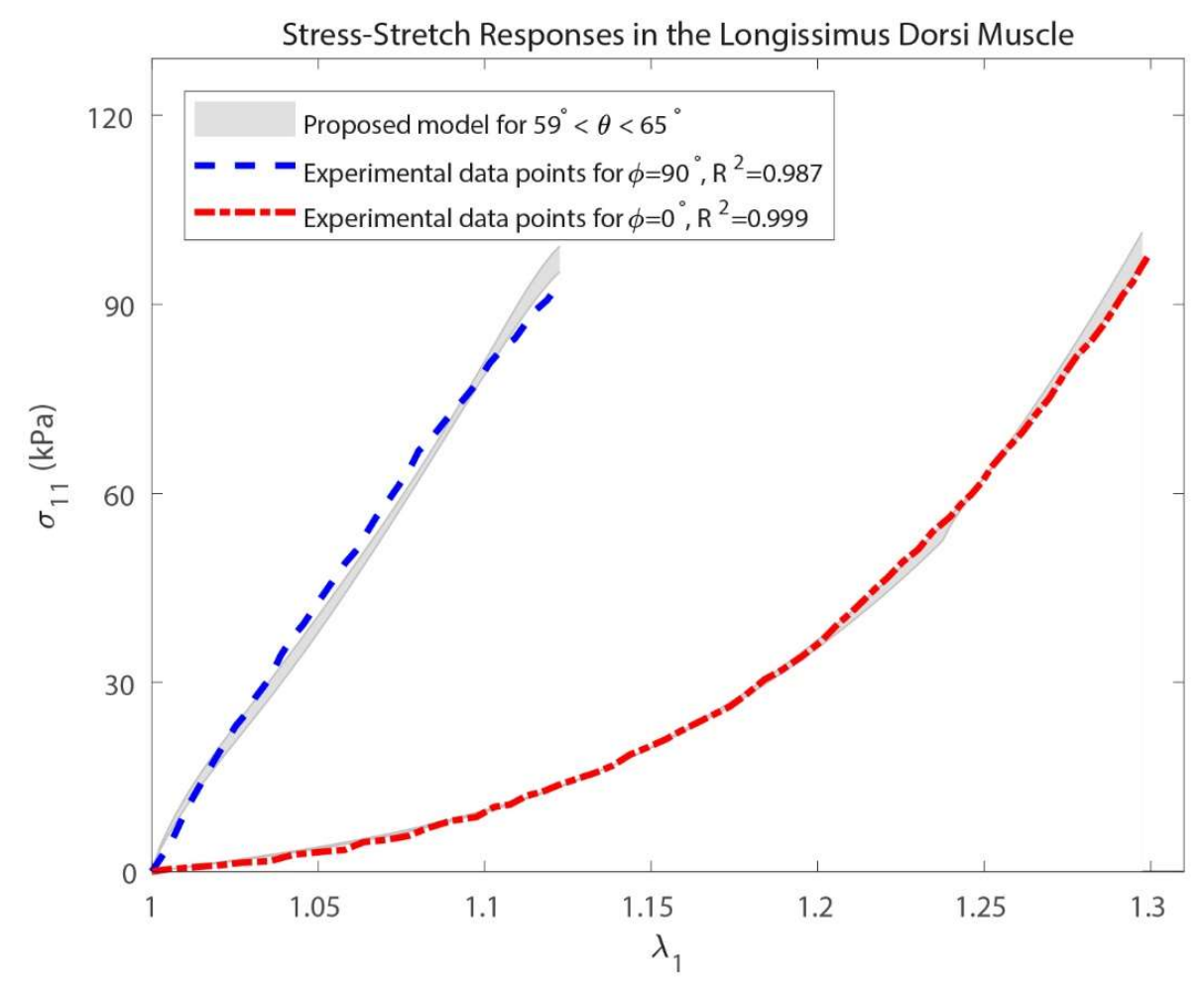

Figure 9. Stress-stretch responses of the pig longissimus dorsi muscle tissue to the uniaxial tension tests in different loading angles with respect to the fiber direction. Also, the ranges of the best approximations of the proposed model for $\phi=0^{\circ}$ and $90^{\circ}$ are depicted as gray bands.

As shown on figure 9, the proposed CL with the reported parameters $c_{1-4}$ has been accurately adapted to fit the experimental data. For $\phi=0^{\circ}$, there is a slight slope change around $\lambda_{1}=1.24$ in the corresponding gray band, which indicates the immediate recruitment of the CFs and their contribution to the resultant stress at $\theta=59^{\circ}$. Also, similar to the explanations provided to interpret the estimated parameters of table 2 , the parameters $c_{1}$ and $c_{2}$ approximately remained constant for the cases of $\theta=63^{\circ}$ and $\theta=65^{\circ}$ in table 3 which indicates that over the ranges of stretch used to estimate the material parameters, there would not be any recruitment in the CFs.

Using the estimated material parameters in table 3 , the stress-stretch behavior of the longissimus dorsi muscle has been predicted at $\phi=30^{\circ}, 45^{\circ}$, and $60^{\circ}$ by implementing the proposed model in equations (7) in the ABAQUS explicit solver. Then, the Cauchy stress at the most central point of the sample is plotted in figure 10. In this figure, the range of the predicted stress is depicted inside the gray bands for this specific range $59^{\circ} \leq \theta \leq 65^{\circ}$. Also, coefficient $R^{2}$ indicates the goodness of the model prediction with respect to the middle curve of each gray band. 


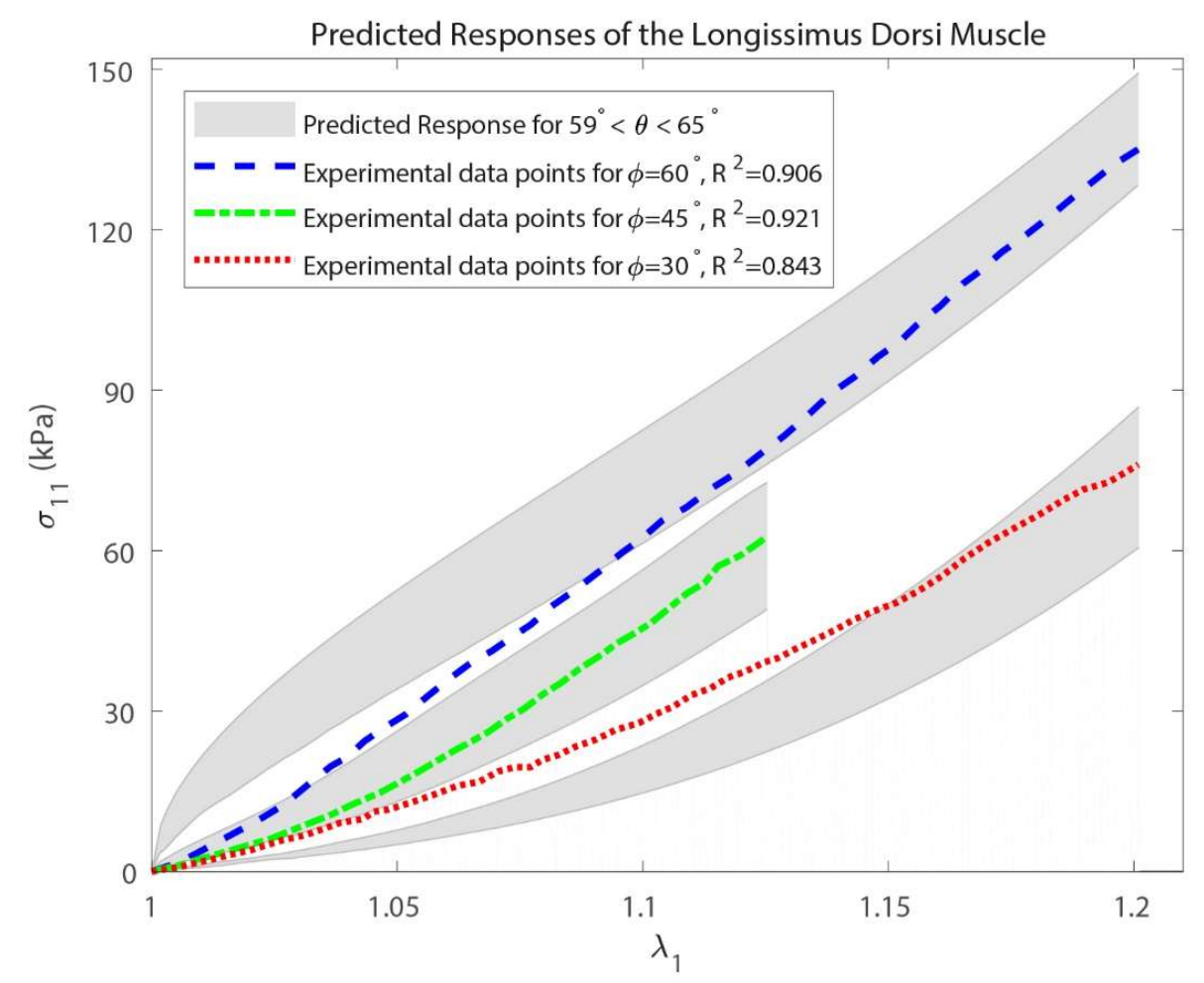

Figure 10. Comparison of the accuracy of the stress-stretch prediction of the proposed CL for the pig longissimus dorsi muscle tissue in loading at different angles $\theta$.

Predicted response for $\phi=60^{\circ}$ begins with a steep slope which is due to the ability of the proposed model to provide a curvature variation for the tissue response. In this case, although the model prediction does not seem to be acceptable for the small values of the stretch, it will be improved if the CL is replaced with another SEF for the CFs contribution, in which the CFs resistance is more smoothly. Providing such a SEF needs including some extra material parameters in the CL. Also, from the plotted bands of figure 10, it is deduced that the mean angle $\theta$ in the longissimus dorsi muscle might be in the range of $59^{\circ} \leq \theta \leq 65^{\circ}$ in the reference configuration.

\subsection{Chicken Pectoralis Muscle}

To complete the evaluation of our model, we use the elastic stress-stretch data of the chicken pectoralis muscle at $\phi=0^{\circ}, 45^{\circ}$, and $90^{\circ}$ (Mohammadkhah et al. 2016). The reported results show that the MF direction is the weakest direction in terms of mechanical resistance and the direction along $45^{\circ}$ with respect to the MF alignment is the stiffest direction. As a result, according to the proposed passive muscle model, obviously, the CFs have a higher contribution to the overall stress for $\phi=90^{\circ}$ than for $\phi=0^{\circ}$. Therefore, the angle $\theta$ must be greater than $45^{\circ}$. With the same policy 
as described above, material parameters $c_{1-4}$ have been estimated for different values of $\theta$ by fitting the Cauchy stress-stretch relationship to the experimental measurements at $\phi=0^{\circ}$ and $90^{\circ}$. The estimated values of the parameters $c_{1-4}$ for $\theta=51^{\circ}, 53^{\circ}$, and $55^{\circ}$ are given in table 4 . For instance, in the case of $\theta=53^{\circ}$, figure 11 shows the approximated Cauchy stress-stretch relationship in the directions parallel and perpendicular to the MF direction.

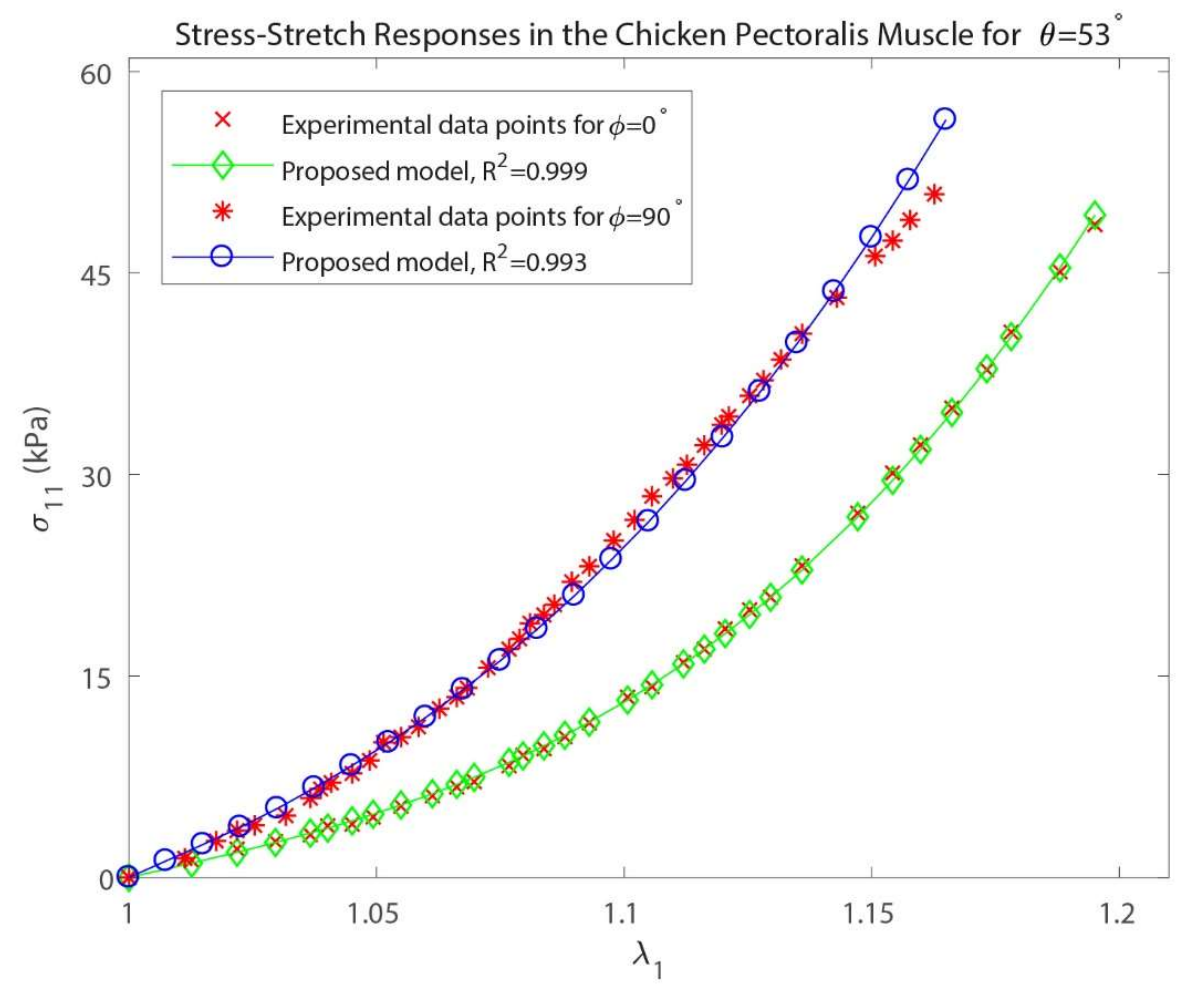

Figure 11. Stress-stretch responses of the chicken pectoralis muscle tissue to the uniaxial tension tests in different loading angles with respect to the MF direction. Also, the best approximation of the proposed model is plotted.

Table 4. Material parameters of the chicken pectoralis muscle tissue estimated for the proposed model.

\begin{tabular}{|c|c|c|c|c|c|c|c|}
\hline & $\begin{array}{c}c_{1} \\
(\mathrm{kPa})\end{array}$ & $\begin{array}{c}c_{2} \\
(\mathrm{kPa})\end{array}$ & $\begin{array}{c}c_{3} \\
(\mathrm{kPa})\end{array}$ & $\begin{array}{l}c_{4} \\
(-)\end{array}$ & $\begin{array}{c}k \\
(\mathrm{kPa})\end{array}$ & $\begin{array}{l}R^{2} \text { for } \\
\phi=0^{\circ}\end{array}$ & $\begin{array}{c}R^{2} \text { for } \\
\phi=90^{\circ}\end{array}$ \\
\hline$\theta=51^{\circ}$ & 84.344 & 9.642 & 460.859 & 2.007 & \multirow{3}{*}{$1 \mathrm{E} 7$} & 0.999 & 0.972 \\
\hline$\theta=53^{\circ}$ & 121.045 & 13.489 & 219.279 & 1.936 & & 0.999 & 0.993 \\
\hline$\theta=55^{\circ}$ & 137.198 & 14.877 & 197.613 & 2.016 & & 0.998 & 0.992 \\
\hline
\end{tabular}

For the chicken pectoralis muscle in which the stiffness of the tissue in the directions of $\phi=$ $0^{\circ}$ and $90^{\circ}$ are much closer to each other, there are two possible deductions. First, the distribution of the CFs is similar to the previous two cases but they must be weaker. Second, their distribution 
is less aligned to the directions perpendicular to the MFs, it means that $\theta$ might have a smaller value. On the other hand, Since chicken pectoralis is stiffer at $\phi=45^{\circ}$, there must be a family of fibers very close to this direction in order to provide such stiffness. Therefore, the second explanation is anticipated to be the case for the chicken pectoralis muscle.

Finally, using these estimated material parameters, the accuracy of the proposed model has been examined at $\phi=45^{\circ}$. In this case, the approximated Cauchy stress-stretch relationship is plotted in figure 12. The FEA results for $\theta=51^{\circ}, 53^{\circ}$, and $55^{\circ}$ at the most central point of the sample are plotted in this figure. It can obviously be seen from figure 12 that the proposed passive muscle model with the tuned material parameters for $\theta=51^{\circ}$ accurately approximates the behavior of the chicken pectoralis muscle. So, it seems that the angle $\theta$ in the reference configuration might be around $\theta=51^{\circ}$ for this muscle. It is remarkable that without any distinction between CFs and MFs, it is impossible to predict such a complex stress-stretch behavior.

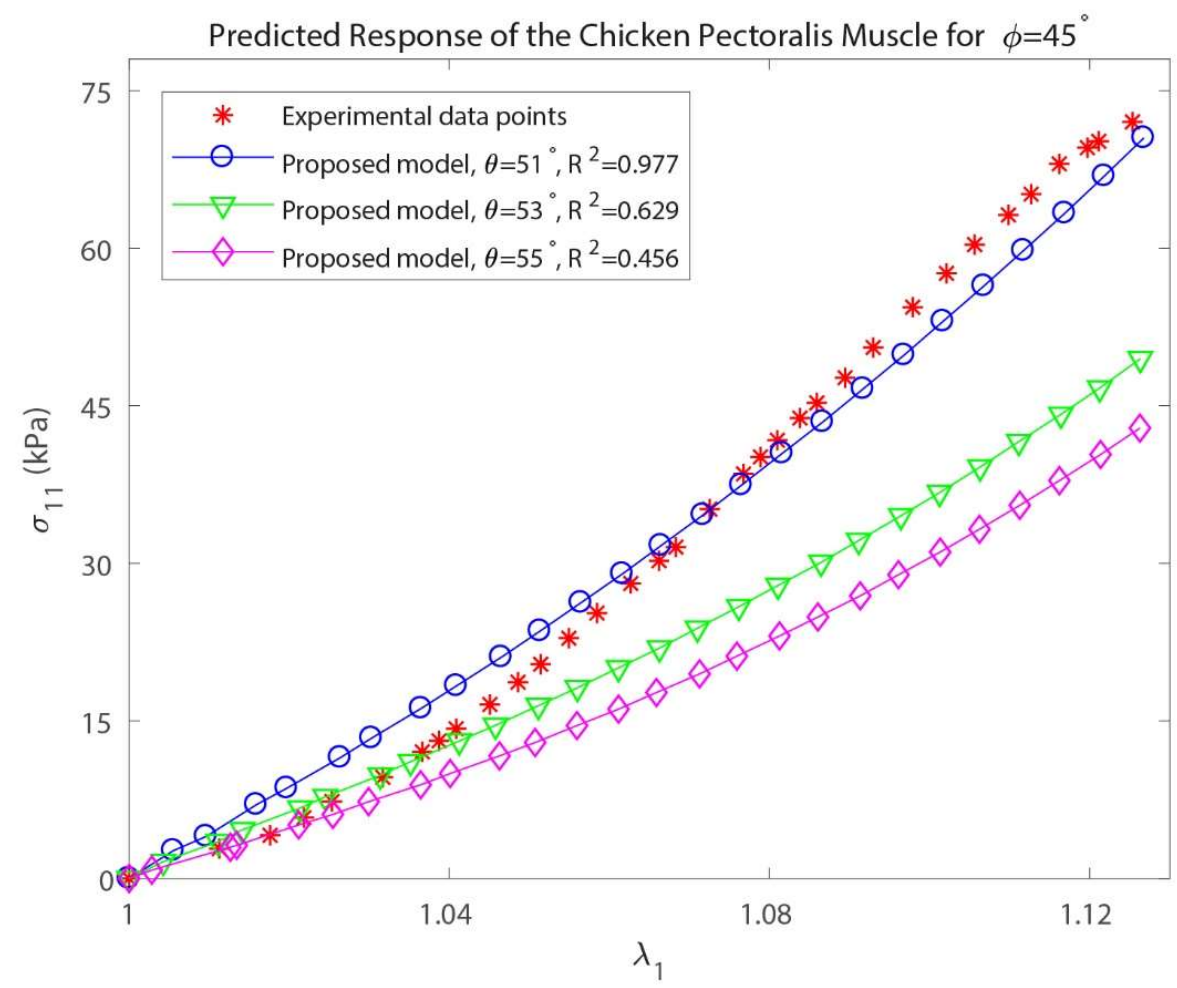

Figure 12. Comparison of the accuracy of the stress-stretch prediction of the proposed CL for the chicken pectoralis muscle tissue in loading at different angles $\theta$. 


\section{Discussions}

The passive mechanical modeling of muscle tissue is very important in various applications of biomechanical studies. However, most of the proposed passive muscle models in the literature cannot rigorously explain all of the observed stress-stretch behaviors in muscle tissue, especially in tissue that amazingly shows less resistance to the applied load along the fiber alignment. In such muscle models that do not distinguish the MFs and the CFs, approximating such an amazing material behavior might need a negative contribution for the anisotropic part of the stress tensor, which violates the convexity condition of the CL. This paper has proposed a new muscle architecture to overcome these drawbacks by taking into consideration the 3-D distribution of the CFs in the muscle tissue. The model is based on the separation of the CFs and their directions from the MFs in some muscles which first was presented by Purslow (1989). Therefore, in such model, the reinforced direction of muscle tissue is not necessarily parallel to the direction of the MFs and it is dependent on the angle $\theta$ between CFs and MFs.

With the aid of an inverse finite element method, an iteration based procedure was proposed for material parameters estimation of muscle tissue under uniaxial tensile tests. Also, it was shown that it is possible to predict the angle $\theta$ between CFs and MFs without any dedicated experimental measurement.

The mechanical properties of the bovine tongue tissue were studied by dissecting some samples from the genioglossus muscle tissue and by measuring its response under uniaxial tensile tests. Among the various muscle groups of the tongue, the genioglossus was chosen because of its ability to provide acceptable macroscopic samples in any direction with respect to the MFs. Samples were stretched in different directions to determine the fiber oriented nature of the genioglossus tissue. To demonstrate the relevance and the adequacy of the proposed passive muscle model, it was appraised in the stress-stretch response prediction of the bovine genioglossus muscle tissue, the pig longissimus dorsi muscle and the chicken pectoralis muscle. A good agreement between FEA results and experimental data points in different angles validates the ability of the proposed muscle model in fully taking into account the nonlinear and orientation dependent nature of muscle tissue.

According to the proposed model, without any dedicated experimental measurement for the angle $\theta$, to completely determine the 3-D behavior of muscle tissue, it is required to perform 
uniaxial tensile tests at least in three different directions. Only for $\theta=0^{\circ}$, the elastic properties can be determined with two tensile tests. In general, there is no restriction for loading directions, but if tests were performed at $\phi=0^{\circ}, 90^{\circ}$, and another arbitrary angle, it would result in less computational costs.

Besides, although the proposed muscle model of this study is based on a simplified distribution of the CFs in muscles, it is the first one that not only can explain the 3-D passive stress response of skeletal muscles but also its transversal stretch approximations are in agreement with some experimental evidence. For instance, when a sample of the longissimus dorsi muscle of pigs is extended to $\lambda_{x}=1.15$ (similar to figure $7 \mathrm{c}$ ), our model prediction for the stretch ratios in the transverse plane zy is $\lambda_{z}=0.881 \lambda_{y}$ while its experimental value is $\lambda_{z}=0.938 \lambda_{y}$ (Takaza et al. 2013). For the chicken pectoralis muscle, when the tissue is extended to $\lambda_{x}=1.2$, the model approximation and experimental measurement are $\lambda_{z}=0.860 \lambda_{y}$ and $\lambda_{z}=0.887 \lambda_{y}$, respectively (Mohammadkhah et al. 2016). Regardless of the differences between the experimental measurements and model predictions, the amount of the shortening in the MFs direction approximated by the proposed model always remains higher than the same property in the direction perpendicular to it $\left(\lambda_{z}<\lambda_{y}\right)$. To provide a more accurate approximation for $\lambda_{z}$ and $\lambda_{y}$, the model must be improved to consider the orientation dependent behavior of muscles in compression which seems to be necessary for modeling the 3-D responses of muscle tissue even in tensile loading. The sliding ability of the actin and myosin filaments in the passive behavior indicates that a muscle tissue is more likely to be shortened along the MF direction than others when the tissue is stretched non-parallel to the MF direction. So, considering the anisotropic nature of muscle tissue in compressive loading will anticipate the improvement of muscle models accuracy.

Moreover, the proposed passive muscle model seems to be capable of predicting the asymmetric behavior of muscle tissue in compressive loading, which means that the reinforced direction in tension is different from the one in compression. It can be explained by the fact that when a muscle tissue is contracted in a certain direction, depending on the angle $\theta$, the CFs may be stretched in transverse directions. Hence, a higher amount of force is required to overcome this extra resistance to stretching in transverse directions. 


\section{Acknowledgments}

This work has been supported by Center for International Scientific Studies and Collaboration (CISSC) and French Embassy in Tehran in the framework of the project Hubert Curien Gundishapur BIOSTOC.

\section{Conflicts of interest}

The authors declare that they have no conflict of interest.

\section{Appendix A}

To show that the final values of the material parameters are independent of a first guess, the identification process has been performed two times for the genioglossus muscle. For the first case, the material parameters with the initial values $\left(a_{i}\right)$ have been provided by the above-described procedure in figure 3 . In the second case, the initial values $\left(b_{i}\right)$ have been chosen by some random values in the range of $0<b_{i} \leq 2 a_{i}$. Table A1 shows the initial values chosen for both cases. Also, figure A1 shows the convergence history of each material parameters through the optimization process. It is obvious that in both scenarios, the material parameters have been converged to the same values which indicate that their final values are independent of the initial guess.

Table A1. Initial values of sets $a_{i}$ and $b_{i}$ which have been used to study their effects on the convergence for the case of $\theta=60^{\circ}$.

\begin{tabular}{|c|c|c|c|c|}
\hline Initial value & $\begin{array}{c}c_{1} \\
(\mathrm{kPa})\end{array}$ & $\begin{array}{c}c_{2} \\
(\mathrm{kPa})\end{array}$ & $\begin{array}{c}c_{3} \\
(\mathrm{kPa})\end{array}$ & $\begin{array}{c}c_{4} \\
(-)\end{array}$ \\
\hline$a_{i}$ & 10.190 & 6.942 & 384.739 & 2.481 \\
\hline$b_{i}$ & 19.192 & 7.084 & 182.382 & 2.925 \\
\hline
\end{tabular}




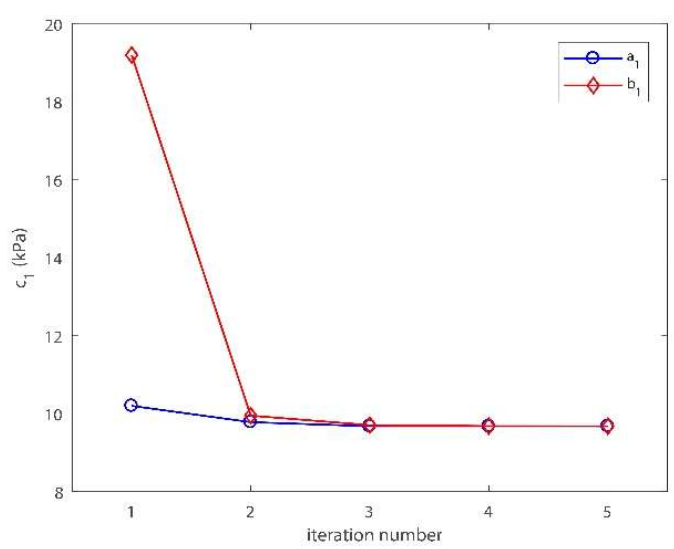

(a)

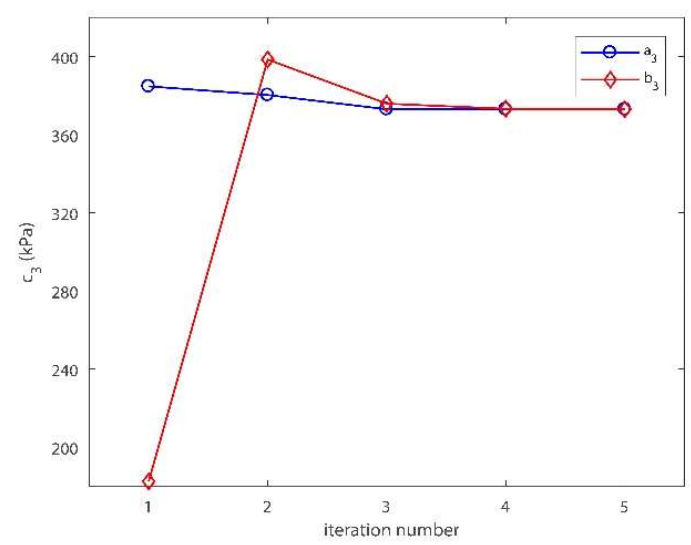

(c)

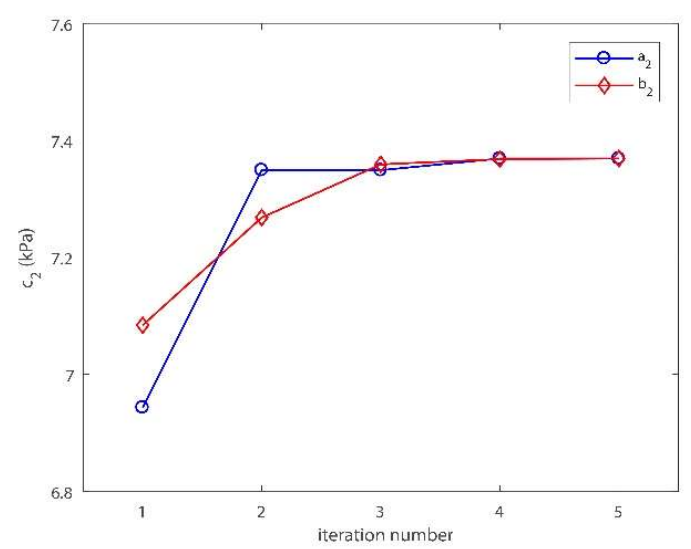

(b)

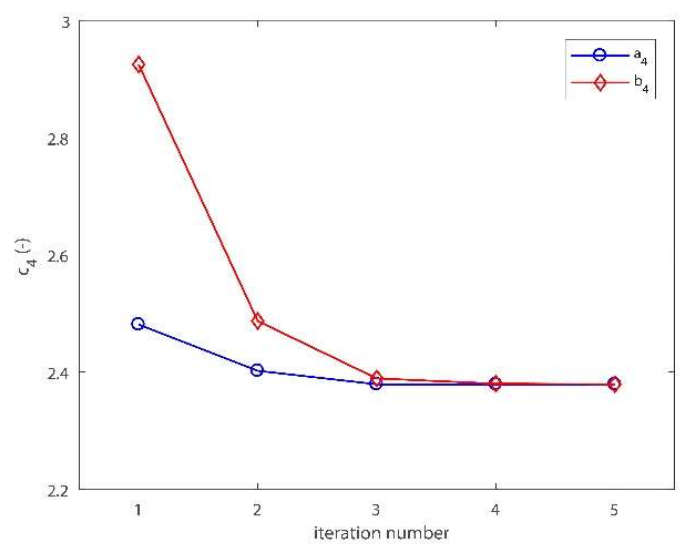

(d)

Figure A1. Convergence study of the material parameters for the case of $\theta=60^{\circ}$ using two different sets of initial values, graphs (a) to (d) show changes in $c_{1}$ to $c_{4}$ in each iteration, respectively.

\section{References}

Blemker, S. S., Pinsky, P. M., \& Delp, S. L. (2005). A 3D model of muscle reveals the causes of nonuniform strains in the biceps brachii. Journal of biomechanics, 38(4), 657-665.

Böl, M., Kruse, R., Ehret, A. E., Leichsenring, K., \& Siebert, T. (2012). Compressive properties of passive skeletal muscle-The impact of precise sample geometry on parameter identification in inverse finite element analysis. Journal of biomechanics, 45(15), 2673-2679.

Böl, M., Ehret, A. E., Leichsenring, K., Weichert, C., \& Kruse, R. (2014). On the anisotropy of skeletal muscle tissue under compression. Acta biomaterialia, 10(7), 3225-3234.

Böl, M., Leichsenring, K., Ernst, M., \& Ehret, A. E. (2016). Long-term mechanical behaviour of skeletal muscle tissue in semi-confined compression experiments. Journal of the mechanical behavior of biomedical materials, 63, 115-124.

Buchaillard, S., Perrier, P., \& Payan, Y. (2009). A biomechanical model of cardinal vowel production: muscle activations and the impact of gravity on tongue positioning. The Journal of the Acoustical Society of America, 126(4), 2033-2051. 
Calvo, B., Ramírez, A., Alonso, A., Grasa, J., Soteras, F., Osta, R., \& Muñoz, M. J. (2010). Passive nonlinear elastic behaviour of skeletal muscle: experimental results and model formulation. Journal of biomechanics, 43(2), 318-325.

Chagnon, G., Rebouah, M., \& Favier, D. (2015). Hyperelastic energy densities for soft biological tissues: a review. Journal of Elasticity, 120(2), 129-160.

Dang, J., \& Honda, K. (2002). Estimation of vocal tract shapes from speech sounds with a physiological articulatory model. Journal of Phonetics, 30(3), 511-532.

Flory, P. J. (1961). Thermodynamic relations for high elastic materials. Transactions of the Faraday Society, 57, 829-838.

Fujita, S., Dang, J., Suzuki, N., \& Honda, K. (2007). A computational tongue model and its clinical application. Oral Science International, 4(2), 97-109.

Gasser, T. C., Ogden, R. W., \& Holzapfel, G. A. (2006). Hyperelastic modelling of arterial layers with distributed collagen fibre orientations. Journal of the royal society interface, 3(6), 15-35.

Gérard, J. M., Ohayon, J., Luboz, V., Perrier, P., \& Payan, Y. (2005). Non-linear elastic properties of the lingual and facial tissues assessed by indentation technique: application to the biomechanics of speech production. Medical engineering \& physics, 27(10), 884-892.

Gérard, J. M.,Perrier, P., Payan, Y., 3D biomechanical tongue modeling to study speechproduction, In: Speech Production: Models, Phonetic Processes, andTechniques, 2006, Psychology Press; New York, 85-102.

Gilbert, R. J., Wedeen, V. J., Magnusson, L. H., Benner, T., Wang, R., Dai, G., ... \& Roche, K. K. (2006). Three-dimensional myoarchitecture of the bovine tongue demonstrated by diffusion spectrum magnetic resonance imaging with tractography. The Anatomical Record Part A: Discoveries in Molecular, Cellular, and Evolutionary Biology, 288(11), 1173-1182.

Gindre, J., Takaza, M., Moerman, K. M., \& Simms, C. K. (2013). A structural model of passive skeletal muscle shows two reinforcement processes in resisting deformation. Journal of the mechanical behavior of biomedical materials, 22, 84-94.

Goldberg, D. E. (2006). Genetic algorithms. Pearson Education India.

Gras, L. L., Mitton, D., Viot, P., \& Laporte, S. (2012). Hyper-elastic properties of the human sternocleidomastoideus muscle in tension. Journal of the mechanical behavior of biomedical materials, 15 , 131-140.

Grasa, J., Sierra, M., Lauzeral, N., Munoz, M. J., Miana-Mena, F. J., \& Calvo, B. (2016). Active behavior of abdominal wall muscles: Experimental results and numerical model formulation. Journal of the mechanical behavior of biomedical materials, 61, 444-454.

Hernández, B., Pena, E., Pascual, G., Rodriguez, M., Calvo, B., Doblaré, M., \& Bellón, J. M. (2011). Mechanical and histological characterization of the abdominal muscle. A previous step to modelling hernia surgery. Journal of the mechanical behavior of biomedical materials, 4(3), 392-404.

Hernández-Gascón, B., Peña, E., Pascual, G., Bellón, J. M., \& Calvo, B. (2014). Can Numerical Modelling Help Surgeons in Abdominal Hernia Surgery?. In Computational Modeling of Objects Presented in Images (pp. 167-185). Springer, Cham.

Holzapfel, G. A. (2006). Determination of material models for arterial walls from uniaxial extension tests and histological structure. Journal of theoretical biology, 238(2), 290-302.

Humphrey, J. D., \& Yin, F. C. P. (1987). On constitutive relations and finite deformations of passive cardiac tissue: I. A pseudostrain-energy function. Journal of biomechanical engineering, 109(4), 298-304.

Martins, J. A. C., Pires, E. B., Salvado, R., \& Dinis, P. B. (1998). A numerical model of passive and active behavior of skeletal muscles. Computer methods in applied mechanics and engineering, 151(3-4), 419-433. 
Mohammadkhah, M., Murphy, P., \& Simms, C. K. (2016). The in vitro passive elastic response of chicken pectoralis muscle to applied tensile and compressive deformation. Journal of the mechanical behavior of biomedical materials, 62, 468-480.

Morrow, D. A., Donahue, T. L. H., Odegard, G. M., \& Kaufman, K. R. (2010). Transversely isotropic tensile material properties of skeletal muscle tissue. Journal of the mechanical behavior of biomedical materials, 3(1), 124-129.

Nie, X., Cheng, J. I., Chen, W. W., \& Weerasooriya, T. (2011). Dynamic tensile response of porcine muscle. Journal of Applied Mechanics, 78(2), 021009.

Parente, M. P. L., Jorge, R. N., Mascarenhas, T., Fernandes, A. A., \& Martins, J. A. C. (2009). The influence of the material properties on the biomechanical behavior of the pelvic floor muscles during vaginal delivery. Journal of biomechanics, 42(9), 1301-1306.

Pioletti, D. P., Rakotomanana, L. R., Benvenuti, J. F., \& Leyvraz, P. F. (1998). Viscoelastic constitutive law in large deformations: application to human knee ligaments and tendons. Journal of biomechanics, 31(8), 753-757.

Purslow, P. P. (1989). Strain-induced reorientation of an intramuscular connective tissue network: implications for passive muscle elasticity. Journal of biomechanics, 22(1), 21-31.

Purslow, P. P. (2010). Muscle fascia and force transmission. Journal of bodywork and movement therapies, 14(4), 411-417.

Rohan, P. Y., Lobos, C., Nazari, M. A., Perrier, P., \& Payan, Y. (2017). Finite element models of the human tongue: a mixed-element mesh approach. Computer Methods in Biomechanics and Biomedical Engineering: Imaging \& Visualization, 5(6), 390-400.

Shall, M. S. (2012). Tongue Biomechanics and Motor Control. In Craniofacial Muscles (pp. 229240). Springer New York.

Schröder, J., \& Neff, P. (2003). Invariant formulation of hyperelastic transverse isotropy based on polyconvex free energy functions. International journal of solids and structures, 40(2), 401-445.

Song, B., Chen, W., Ge, Y., \& Weerasooriya, T. (2007). Dynamic and quasi-static compressive response of porcine muscle. Journal of Biomechanics, 40(13), 2999-3005.

Spencer, A. J. M. (Ed.). (1984). Continuum theory of the mechanics of fibre-reinforced composites (Vol. 282). New York: Springer.

Stavness, I., Hannam, A. G., Lloyd, J. E., \& Fels, S. (2006, July). An integrated dynamic jaw and laryngeal model constructed from ct data. In International Symposium on Biomedical Simulation (pp. 169177). Springer, Berlin, Heidelberg.

Takaza, M., Moerman, K. M., Gindre, J., Lyons, G., \& Simms, C. K. (2013). The anisotropic mechanical behaviour of passive skeletal muscle tissue subjected to large tensile strain. Journal of the mechanical behavior of biomedical materials, 17, 209-220.

Tian, L., Henningsen, J., Salick, M. R., Crone, W. C., Gunderson, M., Dailey, S. H., \& Chesler, N. C. (2015). Stretch calculated from grip distance accurately approximates mid-specimen stretch in large elastic arteries in uniaxial tensile tests. Journal of the mechanical behavior of biomedical materials, 47, 107-113.

Van Loocke, M., Lyons, C. G., \& Simms, C. K. (2006). A validated model of passive muscle in compression. Journal of biomechanics, 39(16), 2999-3009.

Van Loocke, M., Lyons, C. G., \& Simms, C. K. (2008). Viscoelastic properties of passive skeletal muscle in compression: stress-relaxation behaviour and constitutive modelling. Journal of biomechanics, 41(7), 1555-1566.

Vogt, F., Lloyd, J. E., Buchaillard, S., Perrier, P.,Chabanas, M., Payan, Y., \& Fels, S. S. (2006, July). Efficient 3D finiteelement modeling of a muscle-activated tongue. In International Symposium on Biomedical Simulation (pp. 19-28). Springer, Berlin, Heidelberg. 
Wheatley, B. B., Odegard, G. M., Kaufman, K. R., \& Donahue, T. L. H. (2016). How does tissue preparation affect skeletal muscle transverse isotropy?. Journal of biomechanics, 49(13), 3056-3060.

Wheatley, B. B., Odegard, G. M., Kaufman, K. R., \& Donahue, T. L. H. (2017). A validated model of passive skeletal muscle to predict force and intramuscular pressure. Biomechanics and modeling in mechanobiology, 16(3), 1011-1022.

Yousefi, A. A. K., Nazari, M. A., Perrier, P., Panahi, M. S., \& Payan, Y. (2018). A visco-hyperelastic constitutive model and its application in bovine tongue tissue. Journal of biomechanics, 71, 190-198. 